\title{
BENDER GROUPS AS STANDARD SUBGROUPS
}

BY

\author{
ROBERT L. GRIESS, JR.( $\left.{ }^{1}\right)$ DAVID R. MASON AND GARY M. SEITZ( $\left.{ }^{2}\right)$
}

To the memory of Vicente Landázuri Narváez

March 12, 1947-January 29, 1976

\begin{abstract}
A subgroup $X$ of a finite group $G$ is called *-standard if $\tilde{X}=X / O(X)$ is quasisimple, $Y=C_{G}(X)$ is tightly embedded in $G$ and $N_{G}(X)=N_{G}(Y)$. This generalizes the notion of standard subgroups.

THEOREM. Let $G$ be a finite group with $O(G)=1$. Suppose $X$ is *-standard in $G$ and $\tilde{X} / Z(\tilde{X}) \simeq L_{2}\left(2^{n}\right), U_{3}\left(2^{n}\right)$ or $\mathrm{Sz}\left(2^{n}\right)$. Assume $X \triangleleft G$. Then $O(X)$

$=1$ and one of the following holds:

(i) $E(G) \simeq X \times X$.

(ii) $X \simeq L_{2}\left(2^{n}\right)$ and $E(G) \simeq L_{2}\left(2^{2 n}\right), U_{3}\left(2^{n}\right)$ or $L_{3}\left(2^{n}\right)$.

(iii) $X \simeq U_{3}\left(2^{n}\right)$ and $E(G) \simeq L_{3}\left(2^{2 n}\right)$.

(iv) $X \simeq \operatorname{Sz}\left(2^{n}\right)$ and $E(G) \simeq \operatorname{Sp}\left(4,2^{n}\right)$.

(v) $X \simeq L_{2}(4)$ and $E(G) \simeq M_{12}, A_{9}, J_{1}, J_{2}, A_{7}, L_{2}(25), L_{3}(5)$ or $U_{3}(5)$.

(vi) $X \simeq \mathrm{Sz}(8)$ and $E(G) \simeq \mathrm{Ru}$ (the Rudvalis group).

(vii) $X \simeq L_{2}(8)$ and $E(G) \simeq G_{2}(3)$.

(viii) $X \simeq \mathrm{SL}(2,5)$ and $G$ has sectional 2 -rank at most 4 .

In particular, if $G$ is simple, $G \simeq M_{12}, A_{9}, J_{1}, J_{2}, R u, U_{3}(5), L_{3}(5), G_{2}(5)$, or ${ }^{3} D_{4}(5)$.
\end{abstract}

1. Introduction. This paper is concerned with those finite groups $G$ containing a standard subgroup of Bender type. Actually we deal with a more general situation as we allow for cores.

A subgroup $X$ of a finite group $G$ is called *-standard if $\tilde{X}=X / O(X)$ is quasisimple, $Y=C_{G}(\tilde{X})$ is tightly embedded in $G$ and $N_{G}(X)=N_{G}(Y)$. A standard subgroup (in the sense of Aschbacher [1]) is clearly *-standard.

We classify finite groups with a *-standard subgroup of Bender type.

TheOREM. Let $G$ be a finite group with $O(G)=1$. Suppose $X$ is *-standard in $G$ and $\tilde{X} / Z(\tilde{X}) \cong L_{2}\left(2^{n}\right), U_{3}\left(2^{n}\right)$, or $\mathrm{Sz}\left(2^{n}\right)$. Assume that $X \sharp G$. Then $O(X)=1$ and one of the following holds:

(i) $E(G) \cong X \times X$.

(ii) $X \cong L_{2}\left(2^{n}\right)$ and $E(G) \cong L_{2}\left(2^{2 n}\right), U_{3}\left(2^{n}\right)$, or $L_{3}\left(2^{n}\right)$.

(iii) $X \cong U_{3}\left(2^{n}\right)$ and $E(G) \cong L_{3}\left(2^{2 n}\right)$.

(iv) $X \cong \mathrm{Sz}\left(2^{n}\right)$ and $E(G) \cong \mathrm{Sp}\left(4,2^{n}\right)$.

Received by the editors July $20,1976$.

AMS (MOS) subject classifications (1970). Primary 20D05, $20 \mathrm{G} 40$.

(')Research supported in part by N.S.F. Grant 76-07280.

(2)Research supported in part by N.S.F. Grant $37982 X 1$. 
(v) $X \cong L_{2}(4)$ and $E(G) \cong M_{12}, A_{9}, J_{1}, J_{2}, A_{7}, L_{2}(25), L_{3}(5)$, or $U_{3}(5)$.

(vi) $X \cong \mathrm{Sz}(8)$ and $E(G) \cong \mathrm{Ru}$ (the Rudvalis group).

(vii) $X \cong L_{2}(8)$ and $E(G) \cong G_{2}(3)$.

(viii) $X \cong \operatorname{SL}(2,5), G$ has sectional 2 -rank at most 4 , so by $[12], E(G) \cong$ $U_{3}(5), L_{3}(5), G_{2}(5)$, or ${ }^{3} D_{4}(5)$.

In particular, if $G$ is simple then $G \cong M_{12}, A_{9}, J_{1}, J_{2}, \mathrm{Ru}, U_{3}(5), L_{3}(5), G_{2}(5)$, or ${ }^{3} D_{4}(5)$.

Let $G$ and $X$ be as in the main theorem with $X \not G$ and let $T_{0}$ be a Sylow 2-subgroup of $Y$. Then, except in cases (v) and (vi), $\left|T_{0}\right|=2$ and $T_{0}$ induces an outer automorphism on $E(G)$. This shows that if $X$ is a standard subgroup and $m\left(C_{G}(Y)\right)>1$, then the conclusion of the main theorem in [3] holds.

The proof of the main theorem involves a "pushing up" procedure. Starting from a Sylow 2-subgroup of $M=N_{G}(X)$, we attempt to find a Sylow 2-subgroup of $G$. At each stage of the procedure there occurs a certain 2-transitive group and this permutation group either has a regular normal 2-subgroup or a normal subgroup isomorphic to $L_{3}(2)$. In all cases except (vi) and (vii) we show that the latter does not occur. When $E(G)=G_{2}(3)$ an $L_{3}(2)$ does occur at the first step in the process, while for $E(G)=\mathrm{Ru}$, a factor of $L_{3}(2)$ occurs in the second step of the process.

The method of proof eventually reduces us to a situation where we may quote a previous characterization theorem. In particular, we will use the work of Goldschmidt [11] and Gilman and Gorenstein [10] in the identification of $E(G)$. In the exceptional cases (v), (vi) and (vii) we also use Aschbacher [2], Dempwolff [6], Assa [4], O'Nan [19], and Harada [14].

The paper is organized so that $\$ 2$ contains preliminary lemmas and $\$ 3$ basic reductions together with the first step of the "pushing up" process. Then $\S \S 4,5,6$ deal with the cases $\tilde{X} \cong L_{2}\left(2^{n}\right), \mathrm{Sz}\left(2^{n}\right), U_{3}\left(2^{n}\right)$, respectively.

2. Preliminaries. The first lemma deals with tightly embedded subgroups in the automorphism group of a Bender group.

(2.1) Let $X$ be a simple Bender group and $X \leqslant Y \leqslant \operatorname{Aut}(X)$. If $X \leqslant F$ and $F$ is a tightly embedded subgroup of $Y$, then one of the following holds:

(i) $F \cap X$ lies in the normalizer of a Sylow 2-subgroup of $X$, has even order, and contains every involution of $F$.

(ii) $F \cap X=1,|F|=2$, and $F$ induces a field automorphism on $X$.

(iii) $F=(F \cap X)\langle t\rangle$, where $|F \cap X|$ is odd, and $t$ induces a field automorphism of order 2 on $X \cong L_{2}(4)$ or $U_{3}\left(2^{n}\right)$. If $X \cong L_{2}(4)$, then $F \cap X \cong$ $Z_{3}$, and if $X \cong U_{3}\left(2^{n}\right), F \cap X \neq 1$ is cyclic of order dividing $2^{n}+1$ and $F \cap X$ centralizes $E\left(C_{X}(t)\right) \cong L_{2}\left(2^{n}\right)$.

Proof. Suppose $t \in F \cap X$ is an involution. Then $t$ is central in a Sylow 
2-subgroup $U$ of $X$, so that $U$ normalizes $F$ and $U(F \cap X)$ is a group. It follows that $U(F \cap X) \leqslant N_{X}(U)$ (see (1.6) of [19]) and, consequently, $F \cap$ $X$ fixes a unique point in the usual 2-transitive permutation representation of $X$. From here we have $F \leqslant N(U)$ as $U$ is the unique Sylow 2-subgroup of the stabilizer of that point. If $F-(F \cap X)$ contained an involution $j$, then $C_{X}(j) \leqslant N(F)$, whereas $j$ must induce a field automorphism of $X$ and $C_{X}(j)$ does not contain a normal Sylow 2-subgroup. We have now verified (i).

Assume now that $|F \cap X|$ is odd and $t$ is an involution in $F$. So $t$ induces a field automorphism on $X$ and, by [22], $X \cong L_{2}\left(2^{n}\right)$ or $U_{3}\left(2^{n}\right)$. So $C_{X}(t) \cong$ $L_{2}\left(2^{n / 2}\right)$ or $L_{2}\left(2^{n}\right)$, respectively, and this group normalizes $F$. Let $V$ be a Sylow 2-subgroup of $C_{X}(t)$. We may assume $C_{X}(t) \cong L_{2}\left(q_{0}\right)$ with $q_{0} \geqslant 4$, as otherwise the result is trivial. So we may write $F \cap X=\left\langle C_{F \cap X}(v): v \in\right.$ $\left.V^{\#}\right\rangle$. If $V \leqslant U \in \operatorname{Syl}_{2}(X)$, then $C_{F \cap X}(v) \leqslant N_{X}(U)$ for each $v \in V^{\#}$. Say $F \cap X \neq 1$. Then from the structure of $N_{X}(U)$ we conclude that $X \cong U_{3}\left(2^{n}\right)$, $n \geqslant 2, F \cap X$ is cyclic of order dividing $2^{n}+1$, and $\left[F \cap X, C_{X}(t)\right]=1$.

In any case $\left[C_{X}(t), F\right] \leqslant F \cap X$, and the above implies $\left[C_{X}(t), F\right]=1$ for $q_{0} \geqslant 4$. This implies that $F=(F \cap X)\langle t\rangle$, and we have either (ii) or (iii).

The next several lemmas deal with 2-groups and their automorphism groups.

(2.2) Let $U$ be a 2-group of order $q^{2}$ and $Y$ a cyclic group of order $q-1$ acting fixed-point-free on $U$. Let $V \triangleleft U$ be $Y$-invariant and such that $U / V$ and $V$ are elementary and equivalent as $F_{2}(Y)$-modules. Then $U$ is abelian.

Proof. Higman [17].

(2.3) Let $U Y$ be as in (2.2) and suppose that $T$ is a 2-group of order $q^{2}$, normalized by $U Y,[T, U] \leqslant T \cap U=V$, and $Y$ is fixed-point-free on $T$. Then one of the following holds:

(i) $[T, U]=1$.

(ii) For any $t \in T-V, u \in U-V,[t, u] \neq 1$.

Proof. This is proved using Lie ring methods. See Dempwolff [6, Lemma 1.1].

(2.4) Let $U$ be a 2-group and $\langle t\rangle \times Y$ acting on $U$ with $t$ an involution and $Y$ cyclic of order $2^{n}-1$. Suppose that $Y$ is regular on $C_{U}(t)^{\#}$. Then one of the following holds:

(i) $U$ is isomorphic to a Sylow 2-subgroup of $L_{3}\left(2^{n}\right)$.

(ii) $U$ is isomorphic to a Sylow 2-subgroup of $U_{3}\left(2^{n}\right)$.

(iii) $U$ is homocyclic of rank $n$ and inverted by $t$.

(iv) $U$ is homocyclic of rank $n$ and each involution in $U\langle t\rangle-U$ is $U$-conjugate to $t$. 
(v) $U$ is elementary abelian of order $2^{2 n}$ and each involution in $U\langle t\rangle-U$ is $U$-conjugate to $t$.

Proof. This is essentially contained in Finkelstein [8, Lemmas 2.1 and 2.2]. However, instead of (iv) and (v) he simply states that $U$ is abelian and each involution in $U\langle t\rangle-U$ is $U$-conjugate to $t$. If $U$ is not homocyclic of rank $n$, then using the action of $Y$ we have $\left|\Omega_{1}(U)\right| \geqslant 2^{2 n}$. As $\left|C_{U}(t)\right|=2^{n}$, this must be an equality. Here the only involutions in $\Omega_{1}(U)\langle t\rangle$ are in $\Omega_{1}(U)$ or in $t C_{U}(t)$ and

$$
t^{U} \cap \Omega_{1}(U)\langle t\rangle=t^{\Omega_{1}(U)}=t C_{U}(t)
$$

Consequently, $U=\Omega_{1}(U)$ and (v) holds.

(2.5) Let $A=A_{1} \times A_{0}$ be an elementary abelian 2-group, $\left|A_{0}\right|=2, A \triangleleft N$, $R=O_{2}(N)$. Suppose also that $N$ contains a cyclic subgroup $K$ which operates regularly on $(R / A)^{\#}$ and on $A_{1}^{\#}$. If $C_{R}\left(A_{0}\right)=A$, then $C_{N}\left(A_{0}\right)$ covers $N / R$.

Proof. Assume $C_{R}\left(A_{0}\right)=A$. Then the action of $K$ on $A$ forces $A_{1}=$ $Z(R)$. Consequently, if $A_{0}=\langle t\rangle$, then $t^{N} \subseteq A_{1} t$. On the other hand, the hypotheses force $|R / A|=\left|A_{1}\right|$ and $t^{R}=A_{1} t$. The result follows.

The following is a useful result of Goldschmidt.

(2.6) Let $T \in \operatorname{Syl}_{2}(G), W$ a weakly closed subgroup of $T$ (with respect to $G$ ), and $A$ an abelian subgroup of $C_{T}(W)$, normal in $T$. Let $\mathcal{S}=\{B \leqslant T: B \leqslant A$, $B$ is conjugate to a subgroup of $A\}$ and set $r=\max \left\{m\left(B / C_{B}(W)\right): B \in \mathcal{S}\right\}$. Then either

(i) $\Omega_{1}(A)$ is strongly closed in $T$ (with respect to $G$ ); or

(ii) there exists $B \in \mathcal{S}$ such that $m(B)+r \geqslant m(A)$; also if $t \in T$ is conjugate to an element of $A$, then $m([A, t]) \leqslant 2 r$, with $m[A, t] \leqslant r$ provided $B / C_{B}(W)$ is elementary for each $B \in \mathcal{S}$.

Proof. Theorem 4 of [11].

The following results are the key to the determination of the Sylow 2-subgroup in a group $G$ satisfying the hypotheses of the main theorem.

We consider groups $G$ satisfying the following.

Hypothesis (*). (1) $R \leqslant G$ is elementary and a Sylow 2-subgroup of a tightly embedded subgroup $K$ of $G$.

(2) There is a subgroup $X \nsubseteq N_{G}(R)$ such that $X \leqslant C_{G}(R)$, and if $U \in$ $\operatorname{Syl}_{2}(X)$, then $U$ is elementary of order $q=2^{n}>4$, and $N_{X}(U) / C_{X}(U)$ is cyclic of order $q-1$ and is regular on $U^{\#}$.

(3) For $S \in \operatorname{Syl}_{2}\left(N_{G}(R)\right)$ with $U \times R=V \unlhd S, S / V$ is faithful on $U$.

(2.7) Assume that $G$ satisfies Hypothesis (*). Then one of the following holds:

(a) $S \in \operatorname{Syl}_{2}(G)$ and $V$ is strongly closed in $S$. 
(b) $S_{1} \in \operatorname{Syl}_{2}(G)$ with $\left|S_{1}: S\right|=2, S_{1}$ acts on $S$ interchanging $U$ and $R$, and $V$ is strongly closed in $S$.

(c) Each of the following holds:

(i) $V-U=\cup\left\{\left(R^{\#}\right)^{g}: g \in G, R^{g} \leqslant V\right\}$.

(ii) $N(V)$ is 2-transitive of degree $q$ on $\Delta=R^{G} \cap V$.

(iii) Either $N(V)^{\Delta}$ contains the Frobenius group of order $q(q-1)$ as a normal subgroup, or $q=8$ and $\left(N(V)^{\Delta}\right)^{\prime} \cong L_{3}(2)$.

Proof. Suppose that $G$ satisfies Hypothesis (*), and that (a) and (b) are false. First note that we may regard $U$ as $\mathbf{F}_{q}$ with $N_{X}(U) / C_{X}(U)$ acting as scalar multiplications and $S / V$ acting as field automorphisms.

We first claim that $S \notin \operatorname{Syl}_{2}(G)$. Otherwise we set $S=T, V=W=A$ in (2.6). As $q>4, V$ is weakly closed in $S$. So the lemma applies and $r<1$. But for $q>4$ this is impossible. Consequently, $S \notin \operatorname{Syl}_{2}(G)$.

As $V$ is weakly closed in $S, N_{G}(S) \leqslant N_{G}(V)$ so $V$ contains more than one conjugate of $R$. Applying (3.6) of [3] (which is independent of any results in this paper) we have (i) and (ii) provided we can show that $R^{G} \cap V \neq$ $\{R, U\}$. So suppose this latter case occurs. Let $y \in N(S)-S$ with $y^{2} \in S$. Then $U=R^{y}$. Set $S_{1}=S\langle y\rangle$. It is easily checked that $V$ is weakly closed in $S_{1}$, and, since $R^{G} \cap V=\left\{R, R^{y}\right\}=R^{S_{1}}$, we have $S_{1} \in \operatorname{Syl}_{2}(G)$. Again we appeal to (2.6) to get a contradiction. At this point we have established (i) and (ii).

Now consider the 2-transitive group $N(V)^{\Delta}$. The stabilizer of $R$ in $N(V)$ will normalize $X$ and, hence, will normalize $N_{X}(U)=N_{X}(V \cap X)$. This implies (using (2) of Hypothesis (*)) that $N(V)^{\Delta}$ satisfies the conditions of Theorem 1.1 of Hering, Kantor and Seitz [16]. We conclude that either $N(V)^{\Delta}$ has a regular normal subgroup, so that (iii) holds, or $N(V)^{\Delta}$ contains $\operatorname{PSL}(2, p)$ acting in its usual 2-transitive representation of degree $p+1$. Suppose the latter case holds. Then $p+1=q=2^{n}$ and $p$ is a Mersenne prime. If we consider $N(V)^{\prime} \cap N(R)$, then this group acts on $U$ inducing a Frobenius group of order $\frac{1}{2}(p-1) p=p(q-2)(q-1)$. This forces $\frac{1}{2}(q-$ 2 ) to divide $n$, and hence $n=3$, completing the proof of (iii).

(2.8) Suppose that $G$ satisfies Hypothesis (*), $V$ is not strongly closed in a Sylow 2-subgroup of $G$, and that conditions (i)-(iii) of (2.7) hold with $(N(V))^{\Delta}$ containing a regular normal subgroup. Let $D$ be a 2-complement of $N_{X}(U)$. Then there is a Sylow 2-subgroup $V_{1}$ of $O_{2^{\prime}, 2}(N(V))$ and a $2^{\prime}$-group $D_{1}$ with the following properties:

(a) $S D_{1} \leqslant N\left(V_{1}\right), V_{1} S \in \operatorname{Syl}_{2}(N(V))$, and $D_{1}$ induces $D$ on $V$.

(b) $V_{1}=U_{1} R$ with $U_{1} \cap R=1$, where $U_{1}=\left[D_{1}, V_{1}\right]$.

(c) $U<U_{1}$, and $U_{1} / U$ and $U$ are equivalent $F_{2}\left(D_{1}\right)$-modules.

Proof. The existence of $V_{1}$ and $D_{1}$ satisfying (a) is easy. By (2.7)(i) $U$ is 
characteristic in $V$ and we consider $V_{1} / U$. Suppose that $\Omega_{1}\left(V_{1} / U\right)=V / U$. Then $V=\Omega_{1}\left(V_{1}\right)$ is weakly closed in $V_{1} S$ and $V_{1} S \in \operatorname{Syl}_{2}(G)$. However, we can now apply (2.6) to conclude that $V$ is strongly closed in $V_{1} S$, contradicting our hypothesis.

So $\Omega_{1}\left(V_{1} / U\right)>V / U$ and, since $D_{1}$ is transitive on $\left(V_{1} / V\right)^{\#}$, each coset of $V / U$ in $V_{1} / U$ contains an involution. Since $D$ centralizes $R$ we must have $D_{1} V_{1}$ centralizing $V / U$. It follows that $V_{1} / U$ is elementary. From here (b) follows as well as the first claim in (c). Finally we get the last statement in (c) by letting $r \in R^{\#}$ and noting that the map $u_{1} U \rightarrow\left[u_{1}, t\right]$ is a $D_{1^{-}}$ homomorphism from $U_{1} / U$ to $U$. The proof is complete.

We next make the observation that the above may be repeated. Namely, suppose that Hypothesis $\left({ }^{*}\right)$ holds for $G, V$ is not strongly closed in a Sylow 2-subgroup of $G$, and $N(V)^{\Delta}$ contains a regular normal subgroup. Choose $D_{1}$ and $V_{1}$ as in (2.8) and consider $G_{1}=\bar{G}=N_{G}(U) / U$. Then for $\bar{g} \in G_{1}, \bar{V}^{g} \leqslant \overline{U_{1} R}$ implies $\bar{V}^{g}=\bar{V}$ or $\bar{V}^{g} \cap \bar{V}=1$. With this we can argue as in (2.7) and (2.8).

Suppose now that the process is repeated until at some stage either the induced 2-transitive group does in fact contain $L_{3}(2)$ as a normal subgroup or the analogue of $V$ in $G_{m}$ is strongly closed in a Sylow 2-subgroup of $G_{m}$ and (a) or (b) of (2.7) holds. Assume that the process terminates in the latter way. Then there is a subgroup $D_{m}$ and a 2-group $V_{m}$ of $G$ such that $\left[D_{m}, V_{m}\right]=U_{m}$ $\geqslant U, R$ normalizes $U_{m}, R \cap U_{m}=1, V_{m}=U_{m} R$, each $D_{m}$-composition factor of $U_{m}$ is isomorphic to $U$ and $D_{m}$ induces $D$ on $U$. Also $S \leqslant N\left(U_{m}\right)$ and $S U_{m} \in \operatorname{Syl}_{2}\left(N_{G}\left(V_{m-1}\right)\right)$.

For $r \in R^{\#}, C_{U_{m}}(r)=N_{U_{m}}(R)=U$, so $U_{m}$ satisfies the hypotheses of (2.4). With this notation we can conclude:

(2.9) Let $G$ satisfy Hypothesis (*) and suppose that the above process does not yield the $L_{3}(2)$ case at any stage. Let $U_{m}$ be as above. Then one of the following holds:

(1) $U_{m}$ is isomorphic to a Sylow 2-subgroup of $U_{3}(q)$ or $L_{3}(q)$ and $U_{m} S \in$ $\operatorname{Syl}_{2}(G)$.

(2) $U<U_{m}$ which is homocyclic of rank $n$ and $U_{m} S \in \operatorname{Syl}_{2}(G)$.

(3) $V$ is strongly closed in a Sylow 2-subgroup of $G$.

(4) $U_{m}=U$ is elementary of order $q^{2}$.

Proof. We may assume $U<U_{m}$, as otherwise (3) follows as in (2.7). Also we assume that $U_{m}$ does not satisfy (v) of (2.4), as otherwise (4) holds. Suppose that $U_{m} S$ is normalized by an element $y \in G-U_{m} S$ and $y^{2} \in$ $U_{m} S$. We first show that $y$ normalizes $U$. If $S=\mathrm{RU}$, then $U=Z\left(U_{m} S\right)$ and this is clear. Suppose $S>R U$. If $S^{\prime} \nless U$, then $S^{\prime}$ contains an element fused to an element in $R^{\#}$. In this case $U=C_{U_{m} S}\left(\left(U_{m} S\right)^{\prime}\right)$ so $y$ normalizes $U$ as claimed. So we suppose that $S^{\prime} \leqslant U$ and, hence, $\left(U_{m} S\right)^{\prime} \leqslant U_{m}$. If $U_{m}$ 
satisfies (i) or (ii) of (2.4) then $\left(U_{m} S\right)^{\prime} \geqslant\left[U_{m}, R\right]$ which is homocyclic of order $2^{2 n}$ and of rank $n$. So here $U=\Omega_{1}\left(\left(U_{m} S\right)^{\prime}\right)$ if $\left(U_{m} S\right)^{\prime}=\left[U_{m}, R\right]$ and $U=Z\left(\left(U_{m} S\right)^{\prime}\right)$ if $\left(U_{m} S\right)^{\prime}>\left[U_{m}, R\right]$. Either way $y \in N(U)$. If $U_{m}$ satisfies (iii) or (iv) of (2.4), then $\left(U_{m} S\right)^{\prime}>U_{m-1}$, so $U=\Omega_{1}\left(\left(U_{m} S\right)^{\prime}\right)$ is normalized by $y$. So in all cases the claim holds.

In particular, $y$ normalizes $C_{U_{m} s}(U)=U_{m} R$. But then $y$ normalizes $\left(U_{m} R\right)^{\prime}$ $=U_{m-1}$. Hence $U_{m} S \notin \operatorname{Syl}_{2}\left(N_{G}\left(U_{m-1}\right)\right)$ so that we are in case (b) of (2.7). But then (4) holds. Thus we may assume $U_{m} S \in \operatorname{Syl}_{2}(G)$. We complete the proof by using (2.4) to get the structure of $U_{m}$.

3. Initial reductions. Let $G$ be a finite group having a ${ }^{*}$-standard subgroup $M_{1}$ such that $\tilde{M}_{1}$ is a Bender group and the conclusions of the main theorem are violated. Choose $|G|$ minimal and $M_{1}$ minimal in the group $G$. Let $M=N_{G}\left(M_{1}\right)$ and $M_{0}=C_{M}\left(M_{1} / O\left(M_{1}\right)\right)$.

Choose $T \in \operatorname{Syl}_{2}(M)$ and set $T_{i}=T \cap M_{i}, i=0,1$. Then $T=T_{1} T_{0} T_{3}$ where $T_{3}$ is cyclic. We set $q=\left|\Omega_{1}\left(\tilde{T}_{1}\right)\right|$, so that $q=2^{m}$ and $\tilde{M}_{1} \simeq L_{2}\left(2^{m}\right)$, $\mathrm{Sz}\left(2^{m}\right)$, or $U_{3}\left(2^{m}\right)$, unless $\tilde{M}_{1}$ is a perfect central extension of $\mathrm{Sz}(8)$, when we set $q=8, m=3$, or $\tilde{M}_{1} \cong \mathrm{SL}(2,5)$, when we set $q=4, m=2$. Let $K_{1}$ be a 2-complement in $N_{M_{1}}\left(T_{0} T_{1}\right)$ and $K=K_{1}^{q-1}$. Finally set $A_{i}=\Omega_{1}\left(T_{i}\right)$ and $A=A_{1} A_{0}$.

The above notation will be maintained throughout the rest of the paper.

(3.1) $M_{1}=\left\langle C_{M_{1}}(t): t \in \operatorname{Inv}\left(T_{0}\right)\right\rangle$.

Proof. $C_{M_{1}}\left(T_{0}\right)$ covers $M_{1} / O\left(M_{1}\right)$. So if $m\left(T_{0}\right)>1$ the result is clear. If $m\left(T_{0}\right)=1$, then it is easy to check that $C_{M_{1}}\left(\Omega_{1}\left(T_{0}\right)\right)$ is a *-standard subgroup, so by minimality of $M_{1}$ we again have the result.

(3.2) $F(G)=1$.

Proof. By hypothesis we have $O(G)=1$. Suppose $O_{2}(G) \neq 1$. For each involution $t \in T_{0}$, the tight embedding property implies that $C_{O_{2}(G)}(t)$ centralizes $M_{1}$. Now (3.1) and the $P \times Q$ lemma imply that $M_{1}<$ $C_{G}\left(O_{2}(G)\right)$. But then $O_{2}(G) \leqslant T_{0}$, so $G \leqslant N\left(O_{2}(G)\right)<N\left(M_{1}\right)$ and $m_{1} \triangleleft$ $G$, a contradiction.

(3.3) There does not exist a normal subgroup $1<N \unlhd G$ such that $N$ has Sylow 2-subgroups of class at most 2.

Proof. If such an $N$ exists, then using (3.2) and the result of Gilman and Gorenstein [10], the structure of $N$ is known. Consideration of the action of $T_{0}$ on $E(N)$ gives a contradiction.

Similarly, we have

(3.4) $G$ does not contain a normal subgroup $1<N \unlhd G$ such that a Sylow 
2-subgroup $S$ of $N$ contains an abelian subgroup $A$ with $A$ strongly closed in $S$ with respect to $N$.

Proof. Use (3.2) and Goldschmidt's theorem [11].

(3.5) (a) $G=\left\langle T_{0}^{G}\right\rangle$.

(b) $\left|G: O^{2}(G)\right|<2$. If the index is 2 , then $G=O^{2}(G) T_{0}$ and $T_{0} \cap O^{2}(G)$ $=1$. In particular, $\left|T_{0}\right|=2$ in this case.

Proof. Set $G_{0}=\left\langle T_{0}^{G}\right\rangle$ and suppose $G_{0}<G$. If $M_{1} \cap G_{0}<Z^{*}\left(M_{1}\right)$, then $M_{1} \cap G_{0}$ is a *-standard subgroup in $N$ and, by minimality of $G$, the structure of $E\left(G_{0}\right)$ is known, from which we have a contradiction.

Suppose that $M_{1} \cap G_{0}<Z^{*}\left(M_{1}\right)$. We claim that $T_{0} \in \operatorname{Syl}_{2}\left(G_{0}\right)$. Otherwise, let $X>T_{0}$ be a 2-subgroup of $G_{0}$ normalizing $T_{0}$. Then $X<N\left(M_{1}\right)$, so $\left[M_{1}, X\right]<M_{1} \cap G_{0}<Z^{*}\left(M_{1}\right)$. But this forces $X<M_{0}$, impossible. Consequently, $T_{0} \in \operatorname{Syl}_{2}\left(G_{0}\right)$ and $G=G_{0} N_{G}\left(T_{0}\right)=G_{0} M$. It follows that $M$ $\cap G_{0}$ is strongly embedded in $G_{0}$, so using Bender's theorem [5] we have a contradiction. This proves (a).

For (b) use the minimality of $G$.

(3.6) There exists $g \in G-M$ such that $1 \neq R=T_{0}^{g} \cap M<T$.

(i) $R \cap T_{0}=1$.

(ii) If $m\left(T_{0}\right)>1$, then $g$ can be chosen such that $R=T_{0}^{g}$.

(iii) If $|R|>2$, then $\Omega_{1}(R) \leqslant \Omega_{1}\left(T_{1}\right) T_{0}$.

(iv) If $m\left(T_{0}\right)>1$, then $T_{0}$ is elementary abelian.

(v) If $m\left(T_{0}\right) \geqslant 3$, then $R=T_{8}^{g}$ for all such $g$ :

Proof. If $m\left(T_{0}\right)=1$, then we apply (3.2) and the $Z^{*}$-theorem of Glauberman. Also, in any case, (i) follows from the tight embedding property. We now assume that $m\left(T_{0}\right)>1$.

At this point we apply the work of Aschbacher [1]. Theorems 1 and 3 of [1] apply directly, while the proof of Theorem 2 carries over with just one change. Namely at a certain point Aschbacher uses $\left[M_{0}, M_{0}^{8}\right] \neq 1$ for any $g \in G$ and his Hypotheses II to conclude that (iii) holds. However, in our case, (iii) follows as in the proof of (2.1). So we may apply the theorems in [1] to obtain (3.6) in the case $m\left(T_{0}\right)>1$.

(3.7) Suppose that $m\left(T_{0}\right)>1$. Then:

(i) There is no subgroup $G_{0}<G$ such that $T<G_{0}, M_{1}=O\left(M_{1}\right)\left(M_{1} \cap G_{0}\right)$, and $M_{1} \cap G_{0}$ is $a^{*}$-standard subgroup of $G_{0}$, but $M_{1} \cap G_{0} \not G_{0}$.

(ii) $O(M)=1$.

Proof. Suppose that $m\left(T_{0}\right)>1$. First we show that (i) implies (ii). So assume (i) to hold, but (ii) false. Let $p$ be a prime divisor of $|O(M)|$ and $P_{0}$ a $T$-invariant Sylow $p$-subgroup of $O(M)$. Extend $P_{0}$ to a $T$-invariant Sylow 
p-subgroup, $P$, of $M_{0} \cap C\left(T_{0} T_{1} O(M) / O(M)\right)$. As $\left[M_{1}, P\right] \leqslant\left[M_{1}, M_{0}\right] \leqslant$ $O(M), N_{M_{1}}(P)$ covers $\tilde{M}_{1}$.

Let $g \in G-M$ be as in (3.6)(ii). Since $P=\left\langle C_{P}(t): t \in\left(T_{0}^{g}\right)^{\#}\right\rangle, P \leqslant M^{g}$. It is easily checked that if $\tilde{M}_{1} \neq L_{2}(4)$, then $T_{1} T_{0} / T_{0}$ is the unique group of its isomorphism type in $T / T_{0}$. Applying this to $T^{8} / T_{8}^{8}$ we have $T_{1} T_{0} \cong$ $\left(T_{1} T_{0}\right)^{g}<M_{1}^{g} M_{0}^{g}$ and the structure of $M_{1}^{g}$ forces $P<M_{0}^{g}$. If $\tilde{M}_{1} \cong L_{2}(4)$ or SL(2,5), this also holds, so in all cases $N_{M \beta}(P)$ covers $\tilde{M}_{1}^{8}$. Setting $G_{0}=$ $N_{G}(P)$ it is easily checked that $M_{1} \cap G_{0}$ is ${ }^{*}$-standard in $G_{0}$. So it suffices to prove (i).

We apply induction to $G_{0} / O\left(G_{0}\right)$. Since $m\left(T_{0}\right)>1$, we must be in case (v) or (vi) of the main theorem. Let $T \subseteq S \in \operatorname{Syl}_{2}\left(G_{0}\right), S_{0} \in \operatorname{Syl}_{2}\left(N_{G}(S)\right)$. First, assume $S \subset S_{0}$.

If $G_{0} / O\left(G_{0}\right) \cong A_{9}$ or $S_{9}$, then $T_{0} \sim T_{1}$ in $G_{0}$ and $Z_{2}(S)$ is a klein group which we may take to be $\langle t\rangle \times\left\langle t^{s}\right\rangle$ for $t \in T_{0}$ and $s \in S$. As $t t^{s} \in Z(S)$, $S_{0}=S C_{S_{0}}(t)$, a contradiction.

Suppose that $G_{0} / O\left(G_{0}\right) \cong J_{2}$ or Aut $\left(J_{2}\right)$. Again we check centralizers to see that for each $t \in T_{0}^{\#} t^{S_{0}} \subseteq t^{G_{0}}$. Using the results in [13] we see that $S$ contains precisely 8 conjugates of $T_{0}$ and $t^{G_{0}} \cap S$ is contained in the union of those conjugates. As $S$ is transitive on $T_{0}^{G_{0}} \cap S$, the tight embedding property gives $S_{0}<S N\left(T_{0}\right)$, and again we have a contradiction.

Next suppose that $\tilde{G}_{0}=G_{0} / O\left(G_{0}\right) \cong \operatorname{Aut}\left(M_{12}\right)$. Then $T_{0} \cap G_{0}^{\prime}=\langle t\rangle$ for some involution $t$ and $C_{\tilde{G}_{0}}(t) \cong S_{5} \times\langle t\rangle$, modulo $O\left(G_{0}\right)$. We have $S \geqslant T$ and $T$ contains a Sylow 2-subgroup of $C_{G_{0}}(t)$, which has the form $T_{1}\langle a\rangle \times$ $\langle t\rangle$ for some involution $a$. Set $A=\langle a\rangle \times Z\left(T_{1}\langle a\rangle\right) \times\langle t\rangle$. Then by Theorem 2 of Harada [15], $G$ is of known type. In particular, $G_{0} O(G)=G$ and certainly $S \in \operatorname{Syl}_{2}(G)$.

Finally we assume that $\bar{G}_{0}=G_{0} / O\left(G_{0}\right) \cong \mathrm{Ru}$. Here we use information about $S$ available in Dempwolff [6]. In his notation $S=V$ and $V$ contains a normal subgroup $W$ such that $F=W^{\prime}=A_{1}$ and $W / A_{1}=W / F$ is elementary of order $2^{8}$ on which $N_{\bar{G}_{0}}(\bar{W} / \bar{F}) \cong \mathrm{GL}(3,2)$ acts irreducibly. Checking centralizers we see that $G_{0}$ controls the fusion of its involution so that $S_{0}$ cannot fuse an involution in $T_{0}^{\#}$ into another $G_{0}$-class of involutions.

Using the argument in Lemma 2.2 of [6] we conclude that $S_{0} \leqslant N_{G}(W)$. So $S_{0}$ permutes the involutions in $W-W^{\prime}=W-F$. However, Lemmas 2.7 and 2.8 of [6] show that $S$ is transitive on $T_{0}^{G} \cap W$. Consequently, $S_{0}=$ $S N_{S_{0}}\left(T_{0}\right)=S$, a contradiction.

Now that $S \in \operatorname{Syl}_{2}(G)$ we can obtain a contradiction by quoting an appropriate characterization theorem giving the structure of $G / O(G)$. For all cases except $G_{0} / O\left(G_{0}\right) \cong \mathrm{Ru}$ we can use the result of Gorenstein and Harada [12]. In the remaining case we quote the recent result of Assa [4]. At this point (3.7) is proved. 
(3.8) $\tilde{M}_{\mathrm{i}} \supsetneqq L_{2}(4)$ or $\operatorname{SL}(2,5)$.

PRoof. If $m\left(T_{0}\right)=1$ and $\tilde{M}_{1} \cong L_{2}(4)$, we can quote Theorem 2 of Harada [15] to get a contradiction. If $m\left(T_{0}\right)=1$ and $\tilde{M}_{1} \cong \operatorname{SL}(2,5)$ let $\langle r\rangle=\Omega_{1}\left(T_{0}\right)$. Then it is easily seen that $r$ is a 2-central involution in $G$. Since $C(t)$ has Sylow 2-subgroups of sectional rank at most 4 we again have a contradiction.

Suppose $m\left(T_{0}\right)>1$. By (3.7) $O(M)=1$, so $O\left(M_{1}\right)=1$. If $M_{1}$ is a standard subgroup of $G$, then we quote Aschbacher [2], while if $M_{1}$ is not standard it is because $E\left(M_{0}\right)$ is conjugate to $M_{1}$ and $E(M)=M_{1} \times M_{1}^{g}$ for some $g \in G$. In particular, $T_{0}$ is a klein group and we can quote Smith [21].

(3.9) $T_{0} \cap T_{1}=1$.

Proof. Suppose false. Then $M_{1} / O\left(M_{1}\right)$ is a perfect central extension of $\mathrm{Sz}(8)$ by $Z_{2}$ or $Z_{2} \times Z_{2}$. First suppose that $m\left(T_{0}\right)=1$. Here $T=T_{0} T_{1}$ and $\Omega_{1}(T)=\Omega_{1}\left(T_{1}\right)$ (as $T_{00}=\Omega_{1}\left(T_{0}\right) \leqslant T_{1}$ and $\left.\Omega_{1}\left(T_{1} / T_{00}\right)=\Omega_{1}\left(T_{1}\right) / T_{00}\right)$. Also $\left[T, \Omega_{1}(T)\right]=T_{0} \cap T_{1}$. Consequently, $N_{G}(T) \leqslant N_{G}\left(T_{0} \cap T_{1}\right)$ and it follows that $T \in \operatorname{Syl}_{2}(G)$. But then $T_{1}$ is a strongly closed subgroup of $T$, contradicting (3.4).

If $m\left(T_{0}\right)>1$, then $T_{0}$ is elementary abelian by (3.6)(iv). Here $\Omega_{1}(T)=$ $\Omega_{1}\left(T_{1}\right) T_{0}$ and the above argument again gives a contradiction.

(3.10) $T \notin \operatorname{Syl}_{2}(G)$.

Proof. If $m\left(T_{0}\right)>1$, then $T_{0}$ is elementary by (3.6), so in all cases $V=\Omega_{1}\left(T_{0} T_{1}\right)=\Omega_{1}\left(Z\left(T_{0} T_{1}\right)\right)$. Suppose that $t \in T-T_{0} T_{1}$ is a conjugate of an involution in $T_{0}$. Then $\tilde{M}_{1} \cong U_{3}(q)$ or $L_{2}(q)$ and $C_{\dot{M}_{1}}(t) \cong L_{2}(q)$ or $L_{2}(\sqrt{q})$, respectively. Moreover, all involutions in $C_{M_{1}}(t) t$ are fused to $t$. Clearly, $C_{M_{1}}(t)^{\prime}<C_{G}(t)^{\prime}$ and, by (3.8), $C_{\dot{M}_{1}}(t)$ is simple so $C_{M_{1}}(t)^{\prime}$ covers $C_{\bar{M}_{1}}(t)$. Now we conclude that some conjugate $t^{8}$ of $t$ induces a nontrivial inner automorphism of $M_{1}$.

Assume that $T \in \operatorname{Syl}_{2}(G)$. If $\tilde{M}_{1} \cong L_{2}(q)$ we use (3.6)(iv) and then (2.6) to conclude that $\Omega_{1}\left(T_{0} T_{1}\right)=T_{1} \Omega_{1}\left(T_{0}\right)$ is strongly closed in $T$. This contradicts (3.4). If $\tilde{M}_{1} \cong \mathrm{Sz}(q)$, then $T=T_{1} \times T_{0}$ and again $\Omega_{1}(T)$ is strongly closed and abelian.

Suppose that $\tilde{M}_{1} \cong U_{3}(q)$ and let $t^{8}$ be as in the first paragraph. The group $M^{8}$ contains $C_{M_{1}}\left(t^{8}\right)$ and $C_{M_{1} / O\left(M_{1}\right)}\left(t^{8}\right)$ has order $(q+1) q^{3}$ or $\frac{1}{3}(q+1) q^{3}$. A 2-complement in $N\left(T_{1}\right) \cap C_{M_{1}}\left(t^{8}\right)$ acts fixed-point-freely on $T_{1} / \Phi\left(T_{1}\right)$, and from the structure of $M^{g}$ we conclude $\Omega_{1}\left(T_{1}\right) \leqslant M$.

In particular, (3.9) implies that $t^{g} \notin T_{1}$. We may assume that $\Omega_{1}\left(T_{0}^{g}\right) \leqslant T$ (this is clear if $m\left(T_{0}\right)=1$, and if $m\left(T_{0}\right)>1$ we use (3.6)(ii) and (2.1)). Let $\Delta=\Omega_{1}\left(T_{0}\right)^{G} \cap V$. Since $N_{M_{1}}(V)$ contains a cyclic group acting regularly on $\Omega_{1}\left(T_{1}\right)$ and since $\Delta \&\left\{\Omega_{1}\left(T_{0}\right), \Omega_{1}\left(T_{1}\right)\right\}$, we argue as in (2.7) to conclude that 
$N(V)^{\Delta}$ is 2-transitive of degree $q$. But $T_{0} T_{1} \leqslant C(V)$ and $\left|T: T_{0} T_{1}\right| \leqslant n<q$. This is a contradiction.

(3.11) Let $T \leqslant S \in \operatorname{Syl}_{2}(G)$. Then $N_{S}(T) \leqslant N_{G}\left(T_{1} T_{0}\right)$.

Proof. Suppose $\tilde{M}_{1} \nRightarrow U_{3}(q)$. Then from (3.6) and (3.8) it is easy to see that $\Omega_{1}\left(T_{0} T_{1}\right)$ is weakly closed in $T$ with respect to $T$ and $T_{1} T_{0}=C_{T}\left(\Omega_{1}\left(T_{0} T_{1}\right)\right)$. If $\tilde{M}_{1} \cong U_{3}(q)$, then we may assume $T>T_{1} T_{0}$. In this case $\Omega_{1}\left(Z\left(\Omega_{1}(T)^{\prime}\right)\right)=J$ $>A_{1}$ is normalized by $N_{S}(T)$, and since $T_{1} T_{0} / J$ is the unique group of its isomorphism type in $T / J$, we have the result.

(3.12) $T_{0}$ is elementary abelian.

Proof. By (3.6)(iv) we may assume that $m\left(T_{0}\right)=1$. Choose $y \in N_{S}(T)-$ $T$. By (3.11) $y \in N\left(T_{1} T_{0}\right)$. Also $T_{0}^{y} \cap T_{0}=1$ and $T_{0} \approx T_{1}$. The KrullSchmidt theorem implies that $T_{0}^{y} \leqslant T_{0} Z\left(T_{1}\right)$, and the result follows from the fact that $Z\left(T_{1}\right)$ is elementary.

(3.13) Let $L=N_{G}(A)$ and $\Delta=A_{0}^{G} \cap A$.

(i) $A-A_{1}=\cup\left\{\left(A_{0}^{\#}\right)^{x}: x \in G, A_{0}^{x} \leqslant A\right\}$ is a disjoint union of $q$ conjugates of $A_{0}$.

(ii) $A_{1}$ is strongly closed in $A$ with respect to $G$.

(iii) $L$ induces a 2 -transitive group on $\Delta$.

Proof. This follows exactly as in the proof of (2.7) once we show $\Delta \neq$ $\left\{A_{0}, A_{1}\right\}$. Suppose that, in fact, $\Delta=\left\{A_{0}, A_{1}\right\}$ and let $y \in N_{S}(T)-S$ for $T \leqslant S \in \operatorname{Syl}_{2}(G)$ (here we use (3.10)). By (3.11) $y \in N\left(T_{1} T_{0}\right) \leqslant N(A)$. As $y \notin T$ we must have $A_{0}^{y}=A_{1}$. If $\tilde{M}_{1} \neq L_{2}(q)$, then $A_{1}<\left(T_{1} T_{0}\right)^{\prime}$ and $A_{0} \leqslant$ $\left(T_{1} T_{0}\right)^{\prime}$, impossible. Therefore $\tilde{M}_{1} \cong L_{2}(q)$. But now $G$ satisfies the conditions of Hypothesis (*) of $\S 2\left(R=T_{0}, K=M_{0}, X=C_{M_{1}}\left(T_{0}\right), U=T_{1}\right)$. So (2.7) implies that $A$ is strongly closed in a Sylow 2-subgroup of $G$, contradicting (3.4).

(3.14) Let $L=N_{G}(A)$ be as in (3.13).

(i) $L^{\Delta}$ contains $O_{2}\left(L^{\Delta}\right)$ as a regular normal subgroup of order $q$.

(ii) $O_{2}\left(L^{\Delta}\right) K_{1}^{\Delta} \unlhd L^{\Delta}$ is a 2-transitive Frobenius group.

Proof. It suffices to show that $L^{\Delta}$ contains a regular normal subgroup. Here we use the proof of (2.7)(iii). If $L^{\Delta}$ does not contain a regular normal subgroup then we must have $\left(L^{\Delta}\right)^{\prime} \cong L_{3}(2)$ and $q=8$. So $\tilde{M} \simeq L_{2}(8)$, Sz(8), or $U_{3}(8)$. Since $L$ has a 7-element acting nontrivially on $A_{1}, L$ induces $L_{3}(2)$ on $A_{1}$.

Let $T<S_{1} \in \operatorname{Syl}_{2}\left(N_{G}(A)\right)$. Then $S_{1}$ contains an element $x$ inducing an automorphism of order 4 on $A$ and satisfying $C_{A}(x) \leqslant A_{1}$. From the Jordan form of $x$ acting on $A$ we conclude that $\left|A_{0}\right|=2$. 
First suppose that $\tilde{M}_{1} \cong \operatorname{Sz}(8)$. The stabilizer $J$ in $L$ of an element $y A \in$ $(T / A)^{\#}$ induces $S_{4}$ on $A_{1}$. But also $J$ must stabilize $[T, y]$, a klein group in $A_{1}$ and $y^{2}$, an involution in $A_{1}$. This is impossible.

Next suppose that $\tilde{M}_{1} \cong U_{3}(8)$. We argue as follows, referring the reader to p. 17 of [9] for the structure of $T_{1}$. Let $z \in C_{A_{1}}\left(x^{2}\right)^{\#} \cap\left[A_{1}, x^{2}\right]$. The square roots in $T_{0} T_{1}$ of $z$ form $9 \operatorname{cosets} x_{i} A, i=1, \ldots, 9$, permuted by $x$. Hence one coset at least, say $x_{1} A$, is fixed by $x$. Then, since $A \leqslant Z\left(T_{0} T_{1}\right), x$ acts on the 4-element set $\left\{\left[x_{1}, x_{i}\right]: i=2, \ldots, 9\right\}$, which an easy computation shows is not the case.

Now assume that $\tilde{M}_{1} \cong L_{2}(8)$. Here $T=T_{0} T_{1}$ is elementary of order $2^{4}$. We claim that $S_{1} \in \operatorname{Syl}_{2}(G)$. For suppose $g \in N\left(S_{1}\right)-S_{1}$ with $g^{2} \in S_{1}$. Then $A^{g} \leqslant S_{1}$, but $A \neq A^{8}$. As $A^{g}$ centralizes $A \cap A^{g},\left|A \cap A^{g}\right|=4$ and $A \cap A^{8}\left\langle A_{1}\right.$. So there is a conjugate $A_{0}^{x}=\left\langle t^{x}\right\rangle \subseteq S_{1}-T$. We may assume $t^{x} A<Z\left(S_{1} / A\right)$. Then $t^{x}$ has two nontrivial Jordan blocks on $A$ and, hence, $C_{S_{1}}\left(t^{x}\right)$ covers $S_{1} / A$. This forces $C_{S_{1}}\left(t^{x}\right)$ to involve $D_{8}$, a contradiction. This proves the claim.

Finally we observe that $S_{1}$ has sectional 2-rank 4 so that the theorem of Gorenstein and Harada [12] gives a contradiction.

We remark that the only groups $G$ in the main theorem satisfying $\left(L^{\Delta}\right)^{\prime} \cong$ $L_{3}(2)$ are those with $G^{\prime} \cong G_{2}(3)$.

Notation (3.15). As in (2.8) we now have the existence of certain subgroups of $L$. Let $L_{0}$ be the subgroup of $L$ stabilizing each element of $\Delta$. Then either $T_{1} T_{0} \in \operatorname{Syl}_{2}\left(L_{0}\right)$ or $\tilde{M}_{1} \cong U_{3}(q),\left|T \cap L_{0}: T_{0} T_{1}\right|=2$, and $T \cap L_{0} \in$ $\operatorname{Syl}_{2}\left(L_{0}\right)$. Choose $R>T \cap L_{0}$, a 2-subgroup of $L$ so that $R^{\Delta}$ is the regular normal subgroup in $L^{\Delta}$. We may assume that $T \leqslant N(R)$. Except in the case $T \cap L_{0}>T_{0} T_{1}$, we may choose a subgroup $D_{1} \leqslant N(R)$ of odd order with $D_{1}$ inducing $K_{1}$ on $T_{0} T_{1}$ and $T_{3} \leqslant N\left(D_{1}\right)$. In those cases set $R_{1}=R$. If $T \cap L_{0}>T_{0} T_{1}$, then $K \leqslant L_{0}$ and $K$ induces a cyclic group of order $q+1$ or $\frac{1}{3}(q+1)$ on $T_{0} T_{1}$ normalized by $\left\langle R, K_{1}\right\rangle$. From here it is easy to see that $R / T_{0} T_{1}$ is elementary and that $R$ contains a subgroup $R_{1}$ of index 2 such that $R_{1}>T_{0} T_{1}, R_{1}$ covers $R / R \cap L_{0}$, and $K_{1}$ normalizes $R_{1}$ module $O\left(L_{0}\right)$. So here we choose $D_{1} \leqslant N\left(R_{1}\right)$ of odd order with $D_{1}$ inducing $K_{1}$ on $T_{0} T_{1}$ and $T_{3} \leqslant N\left(D_{1}\right)$.

Set $R_{0}=\left[R_{1}, D_{1}\right]$.

(3.16) (i) $T_{1} \unlhd R$ and $\left[T_{1}, R_{1}\right] \leqslant A_{1}$.

(ii) $R_{0} \cap A_{0}=1$ and $R_{0} A_{0} T_{3} \in \operatorname{Syl}_{2}(L)$.

(iii) $R_{0}=T_{1} R_{2}$ with $T_{1} \cap R_{2}=A_{1}, R_{2}$ abelian, and $R_{2} / A_{1}$ and $A_{1}$ are isomorphic $\mathrm{F}_{2}\left(D_{1}\right)$-modules.

Proof. We have $A \triangleleft R,[A, R] \leqslant A_{1}$ and $T_{1} T_{0} \triangleleft R$ (as $T_{1} T_{0}=R_{1} \cap$ 
$C(A)$ and $\left.R \leqslant R_{1} T_{3}\right)$. First we show that $T_{1} \triangleleft R$. If $T_{1}=A_{1}$, this follows from (3.13)(ii). Suppose that $\tilde{M}_{1} \cong U_{3}(q)$. As $q>2, K<C(A)$ and $\left[K, T_{1}\right]$ $=T_{1}$. It follows that if $g \in R, A_{0}^{g}<A, T_{1}<M_{1}^{g}$. So $T_{1}=T_{0} T_{1} \cap M_{1}^{g}$ and $g \in N\left(T_{1}\right)$. In particular, $R \leqslant N\left(T_{1}\right)$. Now suppose that $\tilde{M}_{1} \simeq \mathrm{Sz}(q)$. If $R / A$ is not elementary abelian, then since $D_{1}$ is transitive on $(R / T)^{\#}$ and on $(T / A)^{\#}$, we have $\Omega_{1}(R / A)=T / A$. But then $\Omega_{1}(R)=A, R \in \operatorname{Syl}_{2}(G), A$ is strongly closed in $R$, and we contradict (3.4). So $R / A$ is elementary abelian. Let $X / A$ be a $D_{1}$-invariant complement to $T_{0} T_{1} / A$ in $R / A$. We use the action of $D_{1}$ to see that $X / A_{1}$ is elementary abelian. Indeed, if $X / A_{1}$ is not abelian choose $A_{2} / A_{1}$ a hyperplane in $A / A_{1}$ with $X^{\prime} \leqslant A_{2}$. Then since $D_{1}$ is irreducible on $X / A, X / A_{2}$ is extraspecial, contradicting the fact that $n$ is odd. So $X / A_{1}$ is abelian, and from the action of $D_{1}$ we see that $X / A_{1}$ is elementary. Let $x \in X-A_{1}$ and $t \in T_{1}-A_{1}$. Then $x^{t}=x a$ for some $a \in A$ as $R / A$ is abelian. Since $t$ centralizes $x^{2} \in A_{1}$, we must have $x^{2}=$ $(x a)^{2}=x^{2} a^{2}[x, a]=x^{2}[x, a]$. Consequently, $[x, a]=1$ and, as $x \in X-A_{1}$, this forces $a \in A_{1}$. We conclude that $\left[T_{1}, X\right] \leqslant A_{1}$ and $T_{1} \triangleleft R$ as claimed.

Now we complete the proof of (i); that is, we show $\left[T_{1}, R_{1}\right]<A_{1}$. If $T_{1}=A_{1}$ this is obvious. In the other cases we have the result since $D_{1}$ acts irreducibly on $T_{1} / A_{1}$, and $T_{1} / A_{1} \cap Z\left(R / A_{1}\right) \neq 1$.

A previous argument shows that $R_{1} / A$ is elementary if $\tilde{M}_{1} \cong \operatorname{Sz}(q)$. We claim that $R_{1} / A$ is elementary in all cases. If not, then as before $\Omega_{1}\left(R_{1} / A\right)=$ $T_{1} A / A$ and $\Omega_{1}\left(R_{1}\right)=A$. If $\tilde{M}_{1} \cong L_{2}(q)$ it is then easy to see that $A$ is weakly closed in $R T_{3}, R T_{3} \in \operatorname{Syl}_{2}(G)$, and by (2.6) (using $\left.q>4\right) A$ is strongly closed in $R T_{3}$. This contradicts (3.4). Now assume that $\tilde{M}_{1} \cong U_{3}(q)$ and let $D=$ $D_{1}^{q-1}$. Then $D \leqslant L_{0}$ and, as $q>2,\left[D, T_{1}\right]=T_{1}$. But also $\left[D, R_{1}\right] \leqslant T_{1} T_{0}$. Consequently, $\left[R_{1}, D, R_{1}\right] \leqslant\left[T_{1} T_{0}, R_{1}\right] \leqslant A$ and $\left[D, R_{1}, R_{1}\right] \leqslant A$. By the 3-subgroup lemma $\left[R_{1}, R_{1}, D\right] \leqslant A$ and so $R_{1}^{\prime} \leqslant A$. That is, $R_{1} / A$ is abelian and, since $D_{1}$ acts irreducibly on $T_{1} A / A$, we conclude that $\Phi\left(R_{1} / A\right)=1$ and $R_{1} / A$ is elementary.

Choose a $D_{1}$-invariant complement $X / A$ to $T_{1} A / A$ in $R_{1} / A$. We next claim that $X / A_{1}$ is elementary abelian. If not then there is an element $x \in X$ with $x^{2} \in A-A_{1}$. Then $x^{2}$ is $R_{1}$-conjugate to an involution in $A_{0}$. Therefore, $x$ is $R_{1}$-conjugate to a member of $T$, a contradiction.

We now set $R_{2}=\left[D_{1}, X\right]$. Then $A_{1} \leqslant R_{2}$ and $R_{2} \cap A_{0}=1$. As $R_{1} / A_{1}$ is the direct sum of $T_{1} / A, R_{2} / A_{1}$, and $A / A_{1}$, we have $R_{0}=T_{1} R_{2}$. This proves (ii) and the first two parts of (iii). If $t \in A_{0}^{\#}$ then the map $r_{2} A_{1} \rightarrow\left[r_{2}, t\right]$ is a $D_{1}$-isomorphism from $R_{2} / A_{1}$ to $A_{1}$. Apply (2.2) to complete the proof of (3.16).

At this stage we have begun the process of building a Sylow 2-subgroup of $G$. We will complete the proof of the main theorem by taking the cases $\tilde{M}_{1} \cong L_{2}(q), \mathrm{Sz}(q), U_{3}(q)$ separately. 
4. $\tilde{M}_{1} \cong L_{2}(q)$. In this section we assume that $\tilde{M}_{1} \cong L_{2}(q)$. Recall that we are after a contradiction and that, by (3.8), $q>4$.

For this case the group $G$ satisfies the conditions of (*) in $\$ 2$ (setting $R=A_{0}, K=M_{0}, U=A_{1}$ ). We could immediately apply (2.9) provided we knew that at each stage of the process described in $\$ 2$ the 2-transitive group did not involve $L_{3}(2)$. So we first prove this.

Suppose that at some stage $L_{3}(2)$ does occur. Then $\tilde{M}_{1} \cong L_{2}(8)$ and $T=T_{0} \times T_{1}$. By (3.14) $L^{\Delta}$ does contain a regular normal subgroup, so that the difficulty occurs at stage $m+1$ of the inductive process, where $m \geqslant 1$. Consequently, there is a subgroup $U_{m}>A_{1}$ and a subgroup of odd order $D_{m}$, such that $D_{m} A_{0} \leqslant N\left(U_{m}\right), D_{m}$ acts on $A, A_{0}$ as does $D_{1}$, each $D_{m}$ composition factor of $U_{m}$ is isomorphic to $A_{1}$, and if $U_{m-1}=\left[U_{m}, A_{0}\right]$, then $N=N_{G}\left(U_{m-1}\right) \cap N_{G}\left(U_{m} A_{0}\right)$ induces $L_{3}(2)$ on $U_{m} A_{0} / U_{m-1}$, 2-transitive on $\Omega=\left(A_{0} U_{m-1} / U_{m-1}\right)^{N}$. Also $U_{m}$ is normal in $N$ (see (2.7)).

We claim that $U_{m}$ is homocyclic of rank $n,\left|A_{0}\right|=2$, and $A_{0}$ inverts $U_{m}$. To see this, note that for $t \in A_{0}^{\#}, t^{N}$ contains elements in $U_{m} t$. So $t$ inverts elements of $U_{m}$, and, using the action of $D_{m}, t$ inverts an element of each coset of $U_{m-1}$ in $U_{m}$. But now (2.4) implies that $U_{m}$ is abelian, so $t$ inverts $U_{m}$ and $U_{m}$ is homocyclic of rank $n$. As $t \in A_{0}^{\#}$ was arbitrary, $A_{0}=\langle t\rangle$ and we have the claim.

Next note that $t U_{m}=t^{N}$ and the Thompson transfer lemma implies that $t \notin O^{2}(N)$. In particular, $O^{2}(N)$ has index 2 in $N$, is complemented by $\langle t\rangle$, and a Sylow 2-subgroup of $N$ has the form $S=S_{0}\langle t\rangle$, where $S_{0} \cap\langle t\rangle=1$ and $U_{m} \leqslant S_{0} \in \operatorname{Syl}_{2}\left(O^{2}(N)\right)$. Then $S_{0} / U_{m} \cong D_{8}$. As $U_{m}>A_{1}$ has exponent at least $4, U_{m}$ is weakly closed in $S$, and since $U_{m} A_{0}=C_{S}\left(\Omega_{1}\left(U_{m}\right)\right), S \in$ $\operatorname{Syl}_{2}(G)$. In addition it is clear that $t$ does not fuse into $S_{0}$, so by transfer $G$ contains a normal subgroup $G_{0}$ of index 2. Clearly, $S_{0} \in \operatorname{Syl}_{2}\left(G_{0}\right)$. At this point we have the structure of $G_{0}$ by appealing to [15] or to [19]. In either case we have a contradiction.

We may now apply (2.9) to get the subgroup $U_{m}>A_{1}$. Here $S=A_{1} A_{0} T_{3}$ $\in \operatorname{Syl}_{2}(M)$. By (3.13), (2.9)(3) does not hold.

(4.1) $U_{m}$ is not isomorphic to a Sylow 2-subgroup of $U_{3}(q)$ or $L_{3}(q)$.

Proof. Deny. Then $U_{m-1}$ is homocyclic of exponent 4 and, since for each $t \in A_{0}^{\#}, t^{U_{m}}=U_{m-1} t$, we have $t$ inverting $U_{m-1}$. In particular, $A_{0}=\langle t\rangle$. By (2.9) $U_{m} S=U_{m} A_{0} T_{3} \in \operatorname{Syl}_{2}(G)$. Now $A_{0} T_{3}$ is abelian, and if $A_{0} T_{3}$ is cyclic, then we transfer out $A_{0} T_{3}$ and contradict (3.3). So we may assume that $T_{3} A_{0}=T_{3} \times A_{0}$ and $T_{3} \neq 1$. Each involution in $T_{3} U_{m}-U_{m}$ centralizes a homocyclic subgroup of order $q=4^{n / 2}$ and rank $n / 2$ in $U_{m-1}$. Each involution in $U_{m}$ has centralizer of order at least $q^{2}$. So $t^{G} \cap U_{m} T_{3}=\varnothing$ and $G$ contains a normal subgroup $G_{0}$ of index 2 . 
By (3.3) and transfer we may assume that $x^{G_{0}} \cap U_{m} \neq \varnothing$, where $\langle x\rangle=$ $\Omega_{1}\left(T_{3}\right)$. Say $y=x^{8} \in U_{m}$. Then either $y \in A_{1}$ and $U_{m} \leqslant C(y)$ or $y \in U_{m}-$ $A_{1}, U_{m}$ is isomorphic to a Sylow 2-subgroup of $L_{3}(q)$, and $C_{U_{m}}(y)$ contains an elementary abelian subgroup of order $q^{2}$. However, $t \not t x$ (for the same reason that $t \not x x)$, and it follows that $B=C_{M_{1}}(x) /\langle x\rangle$ is a ${ }^{*}$-standard subgroup in $C_{G}(x) /\langle x\rangle$ with $C(B / O(B)) \cap C_{G}(x)$ having $\langle t, x\rangle /\langle x\rangle$ as Sylow 2-subgroup. From the minimality of $G$ we have a contradiction.

(4.2) $U_{m}$ is not homocyclic.

Proof. Suppose $U_{m}$ is homocyclic. Then (2.9)(2) implies that $A_{1}<U_{m}$ and $S=U_{m} A_{0} T_{3} \in \operatorname{Syl}_{2}(G)$. So $q \geqslant 8$ by (3.8). It is now easy to show that $U_{m}$ is weakly closed in $S$.

We apply (2.6) to the weakly closed subgroup $U_{m}$ of $S$ and its subgroup $A_{1}$ (so $T=S, W=U_{m}, A=A_{1}$ ). Let $r$ be the integer given in (2.6).

As $U_{m} A_{0} \leqslant C\left(A_{1}\right), r \leqslant 1$. But from (2.6)(ii) and the fact that $q>4$ we see that, in fact, $r=0$. By (3.4) $A_{1}$ is not strongly closed in $S$, so there is a conjugate $x \in U_{m}\left(A_{0}^{\#}\right)$ of an involution of $A_{1}$. Say $t \in A_{0}^{\#}$ and $x \in U_{m} t$. Then $x \notin U_{m-1} t=t^{U_{m}}$ and so $t$ must invert $U_{m}$. As $C_{A_{0}}\left(U_{m}\right)=1, U_{m} t$ is the unique coset of $U_{m}$ in $U_{m} A_{0}^{\#}$ that contains involutions not in $A$. Also we note that each element of $U_{m} t-U_{m-1} t$ is conjugate to $x$.

Suppose $U_{m} A_{0} \in \operatorname{Syl}_{2}(G)$. If $U_{m}$ has exponent 4, then $U_{m} A_{0}$ has class 2 , against (3.3). If $U_{m}$ has exponent greater than 4 , then $U_{m-1} A_{0}^{\#}$ consists of involutions so each element of $A_{0}^{\#}$ inverts $U_{m-1}$, forcing $\left|A_{0}\right|=2$. But now we transfer out $A_{0}$ from $G_{0}$ and again contradict (3.3).

Thus we may choose $x \in T_{3}-U_{m} A_{0}$ with $x^{2} \in A_{0}$. $x$ clearly has no conjugates in $U_{m} A_{0}$, and if $x^{2} \neq 1$ then $x^{2}$ is an involution in $A_{0}$ and so has no conjugate in $S-U_{m} A_{0}$ (check centralizers). Hence $x \notin O^{2}(G)$ by transfer. By (3.5)(b), $\left|A_{0}\right|=2, x$ is an involution, and $x t \in O^{2}(G)$, where $A_{0}=$ $\langle t\rangle$. But we can transfer out $x t$ also, a contradiction.

(4.3) $U_{m}$ is not elementary abelian of order $q^{2}$.

Proof. Suppose that $U_{m}$ is elementary abelian of order $q^{2}$. Then $U_{m}=R_{2}$ and, for $a \in A_{0}^{\#}, a R_{2}-a A_{1}$ contains no involutions. So $A=\left\langle A_{0}^{G} \cap R\right\rangle$ and $N_{G}(R) \leqslant N_{G}(A)$. Let $S \in \operatorname{Syl}_{2}(G)$ with $R T_{3} \leqslant S$. Then $N_{S}(R)=R T_{3}$.

Suppose that there are no involutions in $R T_{3}-R$. Then $N_{S}\left(R T_{3}\right) \leqslant N_{S}(A)$ $=R T_{3}$ so $R T_{3} \in \operatorname{Syl}_{2}(G)$. Also $R_{2} \triangleleft R T_{3}$ must be strongly closed in $S$, contradicting (3.4). So we may assume that there is an involution $x \in T_{3} R-$ $R$, and since $R / A$ is a free $\mathrm{F}_{2}(\langle x\rangle)$-module, we may take $x \in N\left(A_{0}\right)$. Let $t \in A_{0}^{\#} \cap C(x)$.

As $q>4, R_{2}$ is weakly closed in $R T_{3}=R_{2} A_{0} T_{3}=S_{0}$. Let $S_{1}=N_{S}\left(R_{2}\right)$. If $S_{1}=S_{0}$, then using (2.6) and (3.4) we obtain a contradiction. So assume $S_{1}>S_{0}$. If $a \in N_{S_{1}}\left(S_{0}\right)-S_{0}$ then $A_{0}^{a} \cap R_{2} A_{0}=1$. As $T_{3} R / R$ is cyclic this 
forces $\left|A_{0}\right|=2$ and we may assume that $T_{3} A_{0}=T_{3} \times A_{0}$ with $T_{3}$ cyclic.

Now, let bars denote images modulo $R_{2}$. Since $C_{\bar{s}_{1}}(\bar{t})=\langle\bar{t}\rangle \times \bar{T}_{3}$, we may apply Lemma 2.20 in [18] to conclude that either (i) $\bar{t} \in Z\left(\bar{S}_{1}\right)$, or (ii) $\bar{S}_{1}$ has a subgroup $\bar{S}_{2}$ of index 2 with $\bar{S}_{2}=\overline{D T}_{3}, \bar{D}=\langle\bar{w}, \bar{t}\rangle$ dihedral (with $(\overline{w t})^{2}=1$ ) and $\bar{T}_{3}$ acting on $\bar{D}$ centralizing $\bar{t}$ and normalizing $\langle\bar{w}\rangle$; also $\left|\bar{T}_{3} \cap \bar{D}\right|=2$ and the involutions $\bar{w}^{i} t$ are fused in $\bar{S}_{2}$. Set $\langle s\rangle=\Omega_{1}\left(T_{3}\right)$. Then $\langle\bar{s}\rangle=Z(\bar{D})$. Let $z \in S_{1}-R_{2}$ be an involution, and suppose $m\left(\left[R_{2}, z\right]\right) \leqslant 2$. Then $\bar{z} \notin$ $C(\bar{t})$, so we are in case (ii) above. If $\bar{z} \in \overline{D T}_{3}$, then $z=\bar{z}_{1} \bar{z}_{2}$ with $z_{1} \in D$, $z_{2} \in T_{3}$, and $\left|z_{2}\right|=4$. But $\bar{z} \sim \overline{z s}$, so $m\left(\left[R_{2}, s\right]\right) \leqslant 4$. Hence $q=16, m\left(\left[R_{2}, z\right]\right)$ $=2$. If $\bar{z} \notin \overline{D T}_{3}$, then $\langle\bar{z}, \bar{t}\rangle \geqslant \bar{D}$ is dihedral of order $2|\bar{D}|$ and, hence, we can write $\bar{s}$ as a $\frac{1}{4}|\bar{D}|$ th power of a product of $\bar{z}$ and a conjugate. In particular, $m\left(\left[R_{2}, s\right]\right) \leqslant 4$, so $q=16$ and $m\left(\left[R_{2}, z\right]\right)=2$. Also, $|\bar{D}|=4$ and so in this case $\overline{D T}_{3}=C_{\bar{S}_{1}}(\bar{t})$ and no involution of $\overline{D T}_{3}$ satisfies $m\left(\left[R_{2}, z\right]\right)<2$. At this point one can argue that $R_{2}$ is weakly closed in $S_{1}$. So $S_{1} \in \operatorname{Syl}_{2}(G)$ and using (2.6) we have $R_{2}$ strongly closed in $S_{1}$. This is a contradiction.

At this stage we have considered all cases of (2.4) and we conclude that there are no counterexamples to the main theorem with $\tilde{M}_{1} \cong L_{2}(q), q=2^{n}$ $>4$.

5. $\tilde{M}_{1} \cong \mathrm{Sz}(q)$. Recall the notation of $\S 3$ and assume $\tilde{M}_{1} \cong \mathrm{Sz}(q) . R \in$ $\operatorname{Syl}_{2}(N(A)), R=T_{1} R_{2} A_{0}, R_{2}$ is abelian, and $\left[D_{1}, R\right]=T_{1} R_{2}$. Let $Y=N_{G}(R)$ $\leqslant N_{G}\left(A_{1}\right)$ (as $A_{1}=Z(R)$ ) and consider the induced group $Y^{*}$ on $\Delta=$ $\left\{\left(A / A_{1}\right)^{Y}\right\}$.

We will obtain a contradiction to the standing assumption that $G$ is a counterexample to the main theorem.

(5.1) Suppose $T_{1}$ is isomorphic to the Sylow 2-subgroup of $\mathrm{Sz}(8)$. Then $\operatorname{Aut}\left(T_{1}\right)$ does not involve $L_{3}(2)$.

Proof. Suppose $X=\operatorname{Aut}\left(T_{1}\right)$ does induce $L_{3}(2)$. Then $\operatorname{Aut}\left(T_{1}\right)$ induces $L_{3}(2)$ on $T_{1} / A_{1}$ and on $A_{1}$, and looking at the action of an element of order 7 in $X$ we see that the representations of $X$ on $T_{1} / A_{1}$ and on $A_{1}$ are contragredient. Choose a basis $x_{1} A_{1}, x_{2} A_{1}, x_{3} A_{1}$ of $T_{1} / A_{1}$ and a klein group $X_{0}<X$ centralizing $\left\langle x_{1} A_{1}, x_{2} A_{1}\right\rangle$. Then $X_{0}$ centralizes $\left\langle x_{1}^{2}, x_{2}^{2}\right\rangle$, whereas $X_{0}$ centralizes no klein group in $A_{1}$. This is a contradiction.

(5.2) If $q=8$ and $T_{0} \cong Z_{2} \times Z_{2}$, then $G \cong \mathrm{Ru}$, the Rudvalis group.

Proof. Dempwolff [6] (see the appendix).

(5.3) $|\Delta|<5 q$.

Proof. By Lemma 1.8 of [6], $R-A_{1}$ contains at most $q\left(2 q\left|A_{0}\right|-\left|A_{0}\right|+q\right.$ -2) involutions. Each conjugate of $A$ contains $q\left|A_{0}\right|-q$ involutions outside 
$A_{1}$, and by the tight embedding property, $A^{8} \neq A$ for $g \in Y$ implies $A^{8} \cap A$ $=A_{1}$. The result follows.

(5.4) $|\Delta|=q$ and $Y^{*}$ is 2-transitive on $\Delta$. Either $Y^{*} \cong L_{3}(2)$ or $Y^{*}$ contains a regular normal subgroup.

Proof. Let $N^{*}$ be a minimal normal subgroup of $Y^{*}$. We note that $|\Delta|=1+k(q-1)$ where $k \geqslant 1$ is an integer. This follows since $D_{1}^{*}$ is semiregular on $\Delta-\left\{A / A_{1}\right\}$. Also $\left|Y^{*}\right|=|\Delta| v$ where $q-1 \mid v$ and $v$ is odd.

We claim that $N^{*} \cong L_{3}(2)$ or $N^{*}$ is a $p$-group for some prime $p$. First note that by (3.3) $R \notin \operatorname{Syl}_{2}(Y)$. So $\left|Y^{*}\right|$ is even. By (5.3) $k \leqslant 5$. Consequently, $k=1,3$, or 5. Suppose that $k=3$ or 5 . Then $8 X\left|Y^{*}\right|$. By Feit and Thompson [7] and Gorenstein and Walter [14], if the claim is false then $N^{*} \cong \operatorname{PSL}\left(2, q_{1}\right)$ for some prime power $q_{1}$. Suppose this occurs. Let $P^{*}$ be a Sylow $p$-subgroup of $Y^{*}$ for a primitive divisor $p$ of $q-1$. If $P^{*} \cap N^{*} \neq 1$, then $N_{N^{*}}\left(P^{*} \cap N^{*}\right)$ has order twice an odd number. This implies that some involution in $Y^{*}$ normalizes a conjugate of $P^{*}$. But $P^{*}$ fixes just one point of $\Delta$, and the stabilizer of this point in $Y^{*}$ has odd order. So $P^{*} \cap N^{*}=1$ and by the Frattini argument $P^{*}$ normalizes a Sylow 2-subgroup of $N^{*}$. But then $P^{*}$ centralizes this subgroup (as $p \neq 3)$ and we again have a contradiction.

Finally consider the case $k=1$. Here $Y^{*}$ is 2-transitive. By Hering, Kantor and Seitz [16] either $N^{*}$ is a $p$-group or $N^{*} \cong \operatorname{PSL}\left(2, q_{1}\right)$ for some $q_{1}$. As in (2.7) we must have $q_{1}=7$ and $q=8$ in the latter case.

\section{(5.5) $Y$ does not induce $L_{3}(2)$ on $R / A_{1}$.}

Proof. Suppose $Y$ induces $L_{3}(2)$ on $R / A_{1}$. Then $q=8$ and, by (5.2), $\left|T_{0}\right|=2$ or 8 . The nontrivial irreducible constituents of $D_{1}$ on $R / A_{1}$ are $T_{1} / A_{1}$ and $R_{2} / A_{1}$. These are inequivalent. Also the irreducible $\mathbf{F}_{2}$-modules of $L_{3}(2)$ have degrees $1,3,3,8$. Suppose that the representation of degree 8 is a $Y$-composition factor on $R / A_{1}$. Since any $\mathrm{F}_{2}$-module affording this representation is injective and projective, $R / A_{1}$ is completely reducible as an $\mathrm{F}_{2}(Y)$ module. But then there is an involution $t \in A_{0}$ such that $A_{1}\langle t\rangle \unlhd Y$. As $t^{G} \cap A_{1}\langle t\rangle=t^{R_{2}} \cap A_{1}\langle t\rangle, Y \leqslant R_{2} C(t)$, a contradiction.

Let $V / A_{1}$ be a minimal normal subgroup of $Y / A_{1}$ contained in $R / A_{1}$. Then $\left|V / A_{1}\right|=2$ or 8 and, as above, the case $\left|V / A_{1}\right|=2$ gives a contradiction. So $\left|V / A_{1}\right|=8$ and from the action of $D_{1}$ on $R / A_{1}$ we have $V=T_{1}$ or $R_{2}$. By (5.1) $V \neq T_{1}$, so $R_{2} \triangleleft Y$.

As $Y^{\Delta} \cong L_{3}(2)$ and $\left|T_{0}\right|=2$ or $8, D_{1}$ must contain an element $g$ with $g$ inducing an element of order 3 on $R$ and $C_{A_{0}}(g) \neq 1$. Say $1 \neq t \in C_{A_{0}}(g)$. Then $t$ normalizes $C_{T_{1} R_{2}}(g)$. Now $C_{T_{1} R_{2}}(g)$ has order 8 as $g$ induces the regular module for $Z_{3}$ on each of $A_{1}, T_{1} / A_{1}$, and $R_{2} / A_{1}$. So $C_{T_{1} R_{2}}(g)=$ $\left\langle t_{1}, r_{2}\right\rangle$ for some $t_{1} \in T_{1}-A_{1}, r_{2} \in R_{2}-A_{1}$. Therefore $\left[r_{2}, t\right]=t_{1}^{2}$. 
First suppose that $R_{2}$ is elementary abelian. Let $x \in Y$ be such that $x^{\Delta}$ inverts $g^{\Delta}$ and $x \in N(\langle g\rangle)$. Then $x \in N\left(C_{R}(g)\right)=\left\langle t_{1}, r_{2}\right\rangle C_{A_{0}}(g)$. As neither $T_{1} t$, nor $R_{2} t$ contain involutions not in $A$, we have $t^{x} \in t_{1} r_{2} t A_{1}$. In particular, $t_{1} r_{2} t$ must be an involution. This forces $1=t_{1}^{2} t_{1}^{2}\left[t_{1}, r_{2}\right]$. So $\left[t_{1}, r_{2}\right]=1$ and, by (2.3), $\left[T_{1}, R_{2}\right]=1$. At this point $Y$ normalizes $C_{R}\left(R_{2}\right)=T_{1} R_{2}$ and, arguing as in (5.1) (choosing bases in $T_{1} R_{2} / R_{2}$ rather than in $T_{1} / A_{1}$ ), we obtain a contradiction.

Thus $R_{2}$ is homocyclic and with $t_{1}, r_{2}$, and $t$ as before, $\left\langle r_{2}\right\rangle=C_{R_{2}}(\langle g\rangle)$ and $t$ inverts $r_{2}$. As $t$ commutes with the action of $D_{1}$ on $R_{2}, t$ inverts $R_{2}$. If $T_{1} \leqslant C\left(R_{2}\right)$, then $T_{1} R_{2}\langle t\rangle$ is the extended centralizer in $R$ of $R_{2}$ and $T_{1} R_{2}$, $T_{1} R_{2}\langle t\rangle \leq Y$. For $y \in Y, t^{y} \in T_{1} R_{2} t$. Also $\left[T_{1}, R_{2}\langle t\rangle\right]=1$ and $t$ inverts $R_{2}$, so $t^{y} \in R_{2} t$. Thus $R_{2} t=t^{Y}$ and $T_{1}=C_{T_{1} R_{2}}\left(\left\langle t^{Y}\right\rangle\right)<Y$. This contradicts (5.1). Therefore $T_{1} \& C\left(R_{2}\right)$ and by (2.3) no element in $T_{1}-A_{1}$ commutes with an element in $R_{2}-A_{1}$. The extended centralizer of $R_{2}$ in $R$ is $R_{2}\langle t\rangle$, so $R_{2}\langle t\rangle \unlhd Y$. Let $J / R_{2}\langle t\rangle \leqslant R / R_{2}\langle t\rangle$ be a minimal normal subgroup of $Y / R_{2}\langle t\rangle$. If $J \leqslant R_{2} A_{0}$, then $A_{0}$ would contain a klein group with each involution inverting $R_{2}$. This is ridiculous. So $Y$ induces $L_{3}(2)$ on $J / R_{2}\langle t\rangle$ and $J=R_{2}\langle t\rangle T_{1}$. As $D_{1}$ has inequivalent representations on $T_{1} / A_{1}$ and on $R_{2} / A_{1}$, the representation of $Y$ on $J / R_{2}\langle t\rangle$ is the contragredient of the representation of $Y$ on $R_{2} / A_{1}$.

We now complete the proof of (5.5) using an argument in Dempwolff [6] (see the end of the proof of Lemma 3.4 in [6]). Let $r_{2} \in R_{2}-A_{1}$ with $a=r_{2}^{2}$ and let $K=C_{Y}(a)$. As the representation of $Y$ on $J / R_{2}\langle t\rangle$ is contragredient to the representation of $Y$ on $A_{1}, K$ fixes no involution in $J / R_{2}\langle t\rangle=$ $T_{1} R_{2}\langle t\rangle / R_{2}\langle t\rangle$. Choose $t_{1} \in T_{1}$ with $\left[t_{1}, r_{2}\right]=a$ and $x \in K$ with $\left(t_{1} R_{2}\langle t\rangle\right)^{x}$ $\neq t_{1} R_{2}\langle t\rangle$. Say $t_{1}^{x}=t_{2} t^{\alpha} r$, with $t_{2} \in T_{1}-A_{1}, \alpha=0$ or 1 , and $r \in R_{2}$. Then we have $a=a^{x}=\left[t_{1}^{x}, r_{2}^{x}\right]$. But $r_{2}^{x} \in r_{2} A_{1}$ as $R_{2}$ is homocyclic and the squaring map is a $Y$-isomorphism from $R_{2} / A_{1}$ to $A_{1}$. So

$$
a=\left[t_{2} t^{\alpha} r, r_{2}^{x}\right]=\left[t_{2}, r_{2}^{x}\right]\left[t^{\alpha}, r_{2}^{x}\right]=\left[t_{2}, r_{2}\right]\left[t^{\alpha}, r_{2}\right] \text {. }
$$

We know $\left[t, r_{2}\right]=a$ and, since $\left[t_{2}, r_{2}\right] \neq 1$, this forces $\alpha=0$ and $\left[t_{2}, r_{2}\right]=a$. But then $t_{2} t_{1}^{-1} \in C_{T_{1}}\left(r_{2}\right) \leqslant A_{1}$, whereas $t_{1} R_{2}\langle t\rangle \neq t_{2} R_{2}\langle t\rangle$. This is the final contradiction.

At this stage we know that $Y^{\Delta}$ contains a regular normal subgroup.

(5.6) $T_{1} R_{2} \triangleleft Y$ and $R_{2} \triangleleft Y$.

Proof. Let $V / A_{1}$ be minimal normal in $Y / A_{1}$ with $V \leqslant R$. If $D_{1} \leqslant$ $C\left(V / A_{1}\right)$, then $V \leqslant A$, which is impossible. Also $D_{1}$ must act irreducibly on $V / A_{1}$, so $V=T_{1}$ or $R_{2}$. Suppose that $V=T_{1}$. If $\left[R_{2}, T_{1}\right]=1$, then $R_{2} A_{0}=$ $C_{R}\left(T_{1}\right) \triangleleft Y$. In this case $R_{2}=R_{2} A_{0}-\left(\cup_{g \in y} A_{8}^{g}\right)^{\#} \triangleleft Y$. Suppose that $\left[R_{2}, T_{1}\right] \neq 1$. Then by (2.3) $x \in T_{1}-A_{1}, y \in R_{2}-A_{1}$ implies that $[x, y] \neq$ 1. Consequently neither $x y$ nor $x y t$ centralizes $x$, where $t \in A_{0}$. So $A=$ 
$C_{R}\left(T_{1}\right) \triangleleft Y$, which is false. So in all cases $R_{2} \triangleleft Y$.

Let $V / R_{2}$ be normal in $Y / R_{2}$, with $V \leqslant R_{2} A_{0}$. Choose $V$ maximal such that $T_{1} V \triangleleft Y$. Say $V>R_{2}$. Then $V=R_{2}\left(A_{0} \cap V\right)$ and $A_{0} \cap V$ is tightly embedded in $Y$. So $V$ contains $q^{2}$ conjugates of $A_{0} \cap V$ and each element of $V-R_{2}$ is an involution. It follows that $\left|A_{0} \cap V\right|=2$ and $A_{0} \cap V=\langle t\rangle$ inverts $R_{2}$. Let $U \leqslant Y$ be a $D_{1}$-invariant Sylow 2-subgroup of $Y$, containing $R$. Then $A_{1}=Z(U)$ and we may assume $T_{1} \leqslant C\left(R_{2}\right)$, for otherwise $T_{1} R_{2}=$ $C_{R}\left(R_{2}\right) \triangleleft Y$. Say $u \in U, x \in T_{1}-A_{1}$, and $x^{u}=x y t$ with $y \in R_{2}$. For any $r \in R_{2}$ we have

$$
[x, r]=[x, r]^{u}=[x y t, r]=[x, r][t, r],
$$

whence $[t, r]=1$. This is certainly false, so $V=R_{2}$ as required.

We conclude that $T_{1} R_{2} \triangleleft Y$, proving the result.

Let $U>R$ be a Sylow 2-subgroup of $Y$, invariant under $D_{1}$. Setting $U_{1}=\left[U, D_{1}\right]$ we have $U=U_{1} A_{0}$ and $U_{1} \cap A_{0}=1$.

(5.7) $R_{2}=Z\left(T_{1} R_{2}\right)$.

Proof. Suppose $\left[T_{1}, R_{2}\right] \neq 1$. Consider the map $u_{1} \rightarrow\left[t, u_{1}\right]$, where $u_{1} \in U_{1}$ and $t \in A_{0}^{\#}$ is fixed. Then considering this map from $U_{1} / T_{1} R_{2}$ to $T_{1} R_{2} / A_{1}$ and noting the map commutes with the action of $D_{1}$, we see that $\left[t, U_{1}\right] A_{1}=$ $T_{1}$ or $R_{2}$ (use the fact that $T_{1} / A_{1}$ and $R_{2} / A_{1}$ are inequivalent irreducible $\mathrm{F}_{2}\left(D_{1}\right)$-modules). If $\left[t, U_{1}\right] A_{1}=T_{1}$, then $U_{1}$ normalizes $T_{1}$. As in (5.6) this forces $C_{R}\left(T_{1}\right)=A$ to be normal in $U$, which is a contradiction.

Consequently, $\left[U_{1}, A_{0}\right]=R_{2}$ and $R_{2} A_{0}-R_{2}=\cup_{g \in Y} A_{0}^{g}$. Then $A_{0}=\langle t\rangle$ inverts $R_{2}$. We note $U_{1} / T_{1} R_{2} \cong R_{2} / A_{1} \cong A_{1}$ as $\mathrm{F}_{2}\left(D_{1}\right)$-modules. Also $U_{1} / R_{2}$ is elementary abelian, as otherwise $T_{1} R_{2} / R_{2}=\Omega_{1}\left(U_{1} / R_{2}\right)$ and, for $x \in T_{1}-$ $R_{2}, x R_{2}=u^{2} R_{2}$ for some $u \in U_{1}$. But then $\left[x, R_{2}\right]=\left[u^{2}, R_{2}\right]=1$, contradicting (2.3). As $T_{1} \oiint U_{1},(2.3)$ implies that $U_{1} / A_{1}$ has derived group $R_{2} / A_{1}$ and for each $t_{1} \in T_{1}-A_{1}$ and $r_{2} \in R_{2}-A_{1}$, there is some element $u \in U_{1}$ with $t_{1}^{u}=t_{1} r_{2} a$ with $a \in A_{1}$. But $u$ centralizes $t_{1}^{2}$, so $t_{1}^{2}=t_{1}^{2} r_{2}^{2}\left[t_{1}, r_{2}\right]$ and $r_{2}^{2}=$ $\left[t_{1}, r_{2}\right]$. So $t_{1}$ inverts $r_{2}$. But $t_{1}$ was arbitrary and, by $(2.3), C_{T_{1}}\left(r_{2}\right)=A_{1}$. This is a contradiction.

(5.8) There are members of $\Delta$ not in $R_{2} A_{0} / A_{1}$. Consequently, $\langle x \in \Delta\rangle=$ $R / A_{1}$.

Proof. Suppose that $\Delta \subseteq R_{2} A_{0} / A_{1}$. If $R_{2}$ is elementary, then there are no involutions in $R_{2} A_{0}-A_{1} A_{0}-R_{2}$, a contradiction. Consequently, $R_{2}$ has exponent 4 and $A_{0}=\langle t\rangle$ inverts $R_{2}$.

Also $Y$ normalizes $C_{R}(\langle\Delta\rangle)=T_{1}$. Consider $N=N_{G}\left(T_{1}\right)$ and apply (2.9) to obtain the structure of a Sylow 2-subgroup $U_{2}\langle t\rangle$ of $N$. By (5.1) $N \cap N(R)$ does not involve $L_{3}(2)$, so $U_{2} / T_{1}$ is homocyclic of rank $n$, elementary of order $q^{2}$, the Sylow 2-subgroup of $L_{3}(q)$, or the Sylow 2-subgroup of $U_{3}(q)$. 
We may assume that $D_{1}$ normalizes $U_{2}$. First we note that $U_{2}$ centralizes $T_{1} / A_{1}$ and $A_{1}$. Consequently, $\Phi\left(U_{2}\right) \leqslant C_{U_{2}}\left(T_{1}\right)=U_{3}$. So $U_{2} / U_{3}$ is elementary abelian. Also $T_{1} \varangle U_{3}$, so we may write $U_{2} / U_{3}=T_{1} U_{3} / U_{3} \times$ $U_{4} / U_{3}$, with $U_{4} D_{1}$-invariant. Each nontrivial $D_{1}$-composition factor of $U_{4}$ is equivalent to $A_{1}$, whereas $T_{1} U_{3} / U_{3}$ is $D_{1}$-equivalent to $T_{1} / A_{1}$, which is not equivalent to $A_{1}$. Consequently, $t$ normalizes $U_{4}$.

By (2.9) $U_{4}$ is elementary of order $q^{2}$, homocyclic of rank $n$, The Sylow 2-subgroup of $U_{3}(q)$, or the Sylow 2-subgroup of $L_{3}(q)$. We conclude that $U_{2} / T_{1}$ is elementary or homocyclic of rank $n$. Also $U_{3} \geqslant R_{2}$. We must have $U_{4} / R_{3}$ of order 1 or $q$.

If $U_{2}\langle t\rangle \in \operatorname{Syl}_{2}(G)$, then we get a contradiction as follows. First we claim that $t^{G} \cap U_{2}=\varnothing$. For suppose $t^{g} \in U_{2}$. As each of $U_{2} / T_{1}$ and $U_{2} / U_{3}$ are abelian we have $U_{2}^{\prime} \leqslant T_{1} \cap U_{3}=A_{1}$. Consequently, $\left|C_{U_{2}}\left(t^{8}\right)\right| \geqslant\left|U_{2}\right| / q$ and it follows that $\left|U_{2}\right|=q^{3}$. Therefore $U_{2}=T_{1} U_{3}=T_{1} R_{2}$. But then $R_{2}<$ $C\left(t^{8}\right)$, which is impossible. This proves the claim.

Now apply the Thompson transfer lemma to conclude $t \notin G^{\prime}$. Clearly $U_{2} \leqslant G^{\prime}$ by the action of $D_{1}$. Notice that $U_{2} / A_{1}$ is abelian, so that $U_{2}$ has class 2 . This is against (3.3).

We now have that $U_{2}\langle t\rangle \notin \operatorname{Syl}_{2}(G)$. Assume also that $U_{4}$ is not abelian. Then for $x \in U_{4}-R_{2}$ and $t_{1} \in T_{1}$,

$$
\begin{aligned}
\left(t_{1} x t\right)^{2} & =t_{1}(x t) t_{1}(x t)=t_{1}^{2}(x t)\left[x t, t_{1}\right](x t) \\
& =t_{1}^{2}(x t)^{2}\left[x t, t_{1}\left[t_{1}, x t\right]\right] \in(x t)^{2} A_{1}
\end{aligned}
$$

because $t_{1}^{2} \in A_{1}$ and $\left[T_{1}, U_{2}\langle t\rangle\right] \leqslant A_{1}$. As $(x t)^{2} \in R_{2}-A_{1}$, so such element is an involution. In particular,

$$
t^{G} \cap U_{2}\langle t\rangle \subseteq T_{1} U_{3}\langle t\rangle=T_{1} R_{2}\langle t\rangle=R .
$$

It is easy to see that there are no involutions in $T_{1}^{\#} R_{2} t$, and the claim above shows that $t^{G} \cap U_{2}=\varnothing$. Consequently, $t^{G} \cap U_{2}\langle t\rangle=R_{2} t=t^{U_{2}\langle t\rangle}$, and it follows that $U_{2}\langle t\rangle \in \operatorname{Syl}_{2}(G)$, a contradiction. Therefore $U_{4}$ is a homocyclic of rank $n$.

Suppose $U_{4}=U_{3} \leqslant C\left(T_{1}\right)$. Let $g \in N\left(U_{2}\langle t\rangle\right)=P$ with $t^{8}=t_{1} u_{4} t, t_{1} \in$ $T_{1}-A_{1}$, and $u_{4} \in U_{4}$. One checks directly that it is not possible for $C\left(t^{8}\right) \cap$ $T_{1} U_{4}$ to cover $T_{1} U_{4} / U_{4}$ and so this is impossible. Therefore for $g \in P$, $t^{8} \in U_{4}\langle t\rangle$ and, hence,

$$
C\left(\left\langle t^{B}\right\rangle\right) \cap T_{1} U_{4}\langle t\rangle=T_{1} \triangleleft B,
$$

a contradiction. Therefore $U_{4}>U_{3} \geqslant R_{2}$.

Now $Z_{2}\left(U_{2}\langle t\rangle\right)=T_{1} R_{2}$ so $P$ normalizes each of $T_{1} R_{2}, R_{2}=Z\left(T_{1} R_{2}\right)$, $T_{1} U_{4}=U_{2}\langle t\rangle \cap C\left(R_{2}\right)$, and $T_{1} U_{3}=\left(T_{1} R_{2}\right) Z\left(T_{1} U_{4}\right)$. Consider $P=$ $N\left(U_{2}\langle t\rangle\right)$. We want to apply (2.9) to $P / T_{1} U_{3}$. First we must show that $P$ does not involve $L_{3}(2)$. 
Suppose that $P$ involves $L_{3}(2)$. The representations of $P$ on $T_{1} R_{2} / R_{2}$ and on $U_{2} / T_{1} U_{3}$ are contragredient. Say $P \leqslant N\left(U_{4}\right)$. Then we argue as in (5.5) to get a contradiction. Namely, choose $u \in U_{4}-U_{3}$ and set $K=C_{P}\left(u U_{3}\right)$. Choose $t_{1} \in T_{1}-A_{1}$ with $\left[t_{1}, u\right]=a$, where $\langle a\rangle=\Omega_{1}(\langle u\rangle)$. As $K$ fixes no involution in $T_{1} R_{2} / R_{2}$, we choose $k \in K$ with $t_{1}^{k} R_{2} \neq t_{1} R_{2}$. Say $t_{1}^{k}=t_{2} r$, $r \in R_{2}$, and $u^{k}=u u_{3}$, with $u_{3} \in U_{3}$. Then

$$
a=a^{k}=\left[t_{1}^{k}, u^{k}\right]=\left[t_{2} r, u u_{3}\right]=\left[t_{2}, u\right] \text {. }
$$

But then $t_{1} t_{2}^{-1} \in C(u)$, contradicting (2.3).

Therefore $P \leqslant N\left(U_{4}\right)$ and we note that this implies $U_{4} \cap U_{4}^{g}=U_{3}$ for $g \in P-N\left(U_{4}\right)$. Otherwise $\left.C\left(U_{4} \cap U_{4}^{g}\right) \geqslant\left\langle U_{4}, U_{4}^{g}\right\rangle\right\rangle U_{4}$, against (2.3). Choose a Sylow 3-subgroup $J$ of $P$ normalized by $t$. Then a Sylow 2-subgroup of $N_{P}(J)$ has the form $Q=\left\langle t_{1}, u_{4}\right\rangle\langle x, t\rangle$, where $t_{1} \in T_{1}-A_{1}, u_{4} \in U_{4}-$ $U_{3}$, and $Q /\left\langle t_{1}, u_{4}\right\rangle$ is a klein group. Modulo $U_{3}$ we must have $u_{4}^{x}=t_{1} u_{4}$ and $t^{x}=t t_{1} u_{4}$ or $t u_{4}$. Say $t^{x}=t t_{1} u_{4}$ modulo $U_{3}$. As $t^{x^{2}} \in t U_{3}$ and $t_{1}^{x} \in t_{1} U_{3}$, we have

$$
t \equiv t^{x^{2}} \equiv\left(t t_{1} u_{4}\right)^{x} \equiv t t_{1} u_{4} t_{1} u_{4} t_{1} \equiv t t_{1} \quad\left(\bmod U_{3}\right),
$$

impossible. Therefore $t^{x} \in t U_{4}$, so $t$ inverts $U_{4}$. Also $\left[t_{1}, u_{4}\right] \in A_{1} \cap\left\langle t_{1}, u_{4}\right\rangle$ $=\left\langle t_{1}^{2}\right\rangle$, so, by (2.3), $t_{1}^{u_{4}}=t_{1}^{-1}$. Now $t^{x}=t u_{4} u_{3}, u_{4}^{x}=t_{1} u_{4} u_{3}^{\prime}$, for $u_{3}, u_{3}^{\prime}$ in $U_{3}$. As $t^{x}$ must invert $u_{4}^{x}$, we compute and get a contradiction. Therefore $P$ does not involve $L_{3}(2)$ and (2.9) applies.

By (2.9) and a previous argument we obtain a $D_{1}$-invariant subgroup $U_{5}>U_{4}$ such that $D_{1}$ is transitive on $\left(U_{5} / \dot{U}_{4}\right)^{\#}$ and $T_{1} U_{5}\langle t\rangle \in \operatorname{Syl}_{2}(P)$. We have $T_{1} R_{2}=\Omega_{1}\left(T_{1} U_{4}\right)$, so $\left[T_{1}, U_{5}\right] \leqslant R_{2}$. If $u \in U_{5}$ and $t_{1} \in T_{1}-A_{1}$, then $t_{1}^{u}=t_{1} r_{2}$ for $r_{2} \in R_{2}$. But $u$ fixes $t_{1}^{2}$, so that $r_{2}^{2}=1$ and $r_{2} \in A_{1}$. Consequently, $u \in N\left(T_{1}\right)$, contradicting $U_{2}\langle t\rangle \in \operatorname{Syl}_{2}\left(N\left(T_{1}\right)\right)$. This completes the proof of (5.8).

(5.9) $R_{2}$ is elementary abelian.

Proof. By (5.8) there are elements $t_{1} \in T_{1}-A_{1}, r_{2} \in R_{2}, t \in A_{0}^{\#}$ with $t_{1} r_{2} t$ conjugate to an involution in $A_{0}$. Say $t^{u}=t_{1} r_{2} t$ for $u \in U_{1}$. Then

$$
T_{1} \cap T_{1}^{u}=C_{U_{1}}(t) \cap C_{U_{1}}\left(t^{u}\right)
$$

As $T_{1}$ centralizes $r_{2} t$, we conclude from the structure of $T_{1}$ that $T_{1} \cap T_{1}^{u} \leqslant$ $A_{1}\left\langle t_{1}\right\rangle$.

Choose $t_{2} \in T_{1}-A_{1}$. Then $t_{2}^{u}=t_{2} d$ for some $d \in R_{2}$. If $t_{2} \notin A_{1}\left\langle t_{1}\right\rangle$, then, by the above, $d \notin A_{1}$. On the other hand $d^{2}=1$. So $R_{2}-A_{1}$ contains involutions and, consequently, $R_{2}$ is elementary.

(5.10) $U_{1} / R_{2}$ is elementary abelian.

Proof. $U_{1} / A_{1}$ is of class at most 2 and $U_{1} / R_{2}$ is abelian by (2.2). For $x, y$ 
in $U_{1},\left[x, y^{2}\right]=[x, y]^{2}$ modulo $A_{1}$, so that $\left[x, y^{2}\right] \in A_{1}$. Therefore

$$
\left[x^{2}, y^{2}\right]=\left[x, y^{2}\right]^{x}\left[x, y^{2}\right]=\left[x, y^{2}\right]^{2}=1 \text {. }
$$

If $U_{1} / R_{2}$ were homocyclic of exponent 4 , then $\Omega_{1}\left(U_{1} / R_{2}\right)=T_{1} R_{2} / R_{2}$ and, by the above, $T_{1}$ is abelian. This is absurd. $U_{1} / R_{2}$ must be elementary.

(5.11) $R_{2}=Z\left(U_{1}\right)$.

Proof. It suffices to prove that $R_{2} \leqslant Z\left(U_{1}\right)$. Suppose otherwise. Consider the semidirect product $R_{2}\left(U_{1} / T_{1} R_{2}\right) D_{1}$, of order $q^{3}\left|D_{1}\right|$. By (5.10) and (2.2), we may apply (2.3). As we are assuming that $R_{2}$ is not central in $U_{1}$, we conclude that if $r_{2} \in R_{2}-A_{1}$ and $u \in U_{1}-T_{1} R_{2}$, then $\left[u, r_{2}\right] \neq 1$.

Recall the proof of (5.9) and the notation. For $t_{2} \in T_{1}-A_{1}\left\langle t_{1}\right\rangle, t_{2}^{u}=t_{2} d$ for $d \in R_{2}-A_{1}$. But $u^{2} \in R_{2}$, so $t_{2}=t_{2}^{u^{2}}=t_{2} d d^{u}$ and $d=d^{u}$. This contradicts the above paragraph.

We can now obtain a contradiction in the case $\tilde{M}_{1} \cong \operatorname{Sz}(q)$. For each involution $t \in A_{0}^{\#}$, both $R_{2}$ and $U_{1} / R_{2}$ are free $\mathrm{F}_{2}\langle t\rangle$-modules.

Suppose $A_{0}=\langle t\rangle$. Then the above implies $t^{G} \cap U=U_{1} t=t^{U}$, and so $U=U_{1}\langle t\rangle \in \operatorname{Syl}_{2}(G)$. By the Thompson transfer lemma $t \notin G^{\prime}$, although $U_{1} \leqslant G^{\prime}$ (use the action of $D_{1}$ ). As $U_{1}$ has class 2 we have a contradiction to (3.3).

Now assume that $\left|A_{0}\right|>2$. Fix $u \in U_{1}-T_{1} R_{2}$ and consider the involutions $t^{u}$ for $t \in A_{0}^{\#}$. We have $t^{u}=t t_{1} r_{2}$ for $t_{1} \in T_{1}-A_{1}, r_{2} \in R_{2}$. As $u^{2} \in R_{2}, t^{u^{2}} \in t A_{1}$. But $t^{u^{2}}=t t_{1} r_{2} t_{1}^{u} r_{2}^{u}$ and $r_{2}=r_{2}^{u}$. So $t_{1} t_{1}^{u} \in A_{1}$ and $T_{1} \cap$ $T_{1}^{u} \geqslant A_{1}\left\langle t_{1}\right\rangle$. On the other hand, $T_{1} \cap T_{1}^{u} \leqslant C\left(A_{0}\right) \cap C\left(A_{0}^{u}\right)$. Choosing $t^{\prime} \neq t$ in $A_{0}^{\#}$ we have $\left(t^{\prime}\right)^{u}=t^{\prime} t_{1}^{\prime} r_{2}^{\prime}$. We claim that $t_{1}^{\prime} A_{1} \neq t_{1} A_{1}$. Otherwise $t t^{\prime} \in A_{0}$ and $\left(t t^{\prime}\right)^{u} \in t t^{\prime} R_{2}$. But as $R_{2}$ is a free $F_{2}\left\langle t t^{\prime}\right\rangle$-module, this implies that $u \in R_{2} C\left(t t^{\prime}\right)$, which is false. Therefore $t_{1}^{\prime} A_{1} \neq t_{1} A_{1}$. Consequently, $t_{1}$ does not centralize $\left(t^{\prime}\right)^{u}$. This is a contradiction, as $t_{1} \in T_{1}^{u}$.

6. $\tilde{M}_{1} \cong U_{3}(q)$. In this section we assume $\tilde{M}_{1} \cong U_{3}(q), q=2^{n} \geqslant 4$, and obtain a contradiction. Let $D_{1}$ be as in $\S 3$ and $D=D_{1}^{q-1}$. Also $R_{0}=T_{1} R_{2}$.

(6.1) $\left|A_{0}\right|=2$.

Proof. Assume $\left|A_{0}\right|>2$. By (3.7) $O(M)=1$. Let $K_{1}$ and $K=K_{1}^{q-1}$ be as in $\S 3$. Then $T_{1} K \leqslant C(A)$ and for each $A_{0}^{g} \leqslant A,\left(C_{M_{1}^{g}}(K)\right)^{\prime} \cong L_{2}(q)$. Set $G_{0}=N_{G}(K)$.

By induction $E\left(G_{0} / O\left(G_{0}\right)\right) \cong A_{9}, J_{2}$, or $M_{12}$. The first case is out by (3.13)(ii). Suppose $E\left(G_{0} / O\left(G_{0}\right)\right) \cong J_{2}$. Then $\left|A_{0}\right|=q=4, R_{2}$ is elementary abelian, and $\left[A_{0} R_{2}, T_{1}\right]=1$ (for this use the 3-subgroups lemma together with $\left[R_{2}, K\right]=1,\left[T_{1}, K\right]=T_{1}$, and $\left.\left[R_{2}, T_{1}\right] \leqslant A_{1}\right)$. Also $G_{0}$ contains an involution $g$ interchanging $A$ and $R_{2}$. (Sylow 2-subgroups of $G_{0}$ are of type 
$L_{3}(4)\langle\sigma\rangle$ where $\sigma$ is a graph-field automorphism. See [13].) Hence $g$ normalizes $O_{2}\left(C_{G}\left(\left\langle A, R_{2}\right\rangle\right)\right)=T_{1}$. Since $[g, K]=1$ and $K$ is irreducible on $T_{1} / A_{1}, g$ centralizes $T_{1} / A_{1}$ and, hence, also $A_{1}$ which is not the case.

Finally we suppose $E\left(G_{0} / O\left(G_{0}\right)\right) \cong M_{12}$. Then $G_{0} / O\left(G_{0}\right) \cup \operatorname{Aut}\left(M_{12}\right)$ and $T_{0} \ll G_{0}$. It follows that there is an involution in $N\left(T_{0}\right) \cap C(K)$ not centralizing $T_{0}$ (e.g. see the table in [3], which gives centralizers of involutions for $\left.\operatorname{Aut}\left(M_{12}\right)\right)$. This is impossible.

(6.2) $\left[T_{1}, R_{2}\right]=1$.

Proof. We have $\left[D, R_{2}\right]=1$ and $\left[D, T_{1}\right]=T_{1}$. So $\left[D, R_{2}, T_{1}\right]=1$ and $\left[R_{2}, T_{1}, D\right] \leqslant\left[A_{1}, D\right]=1$. The 3-subgroups lemma implies the result.

(6.3) (i) $T_{1} R_{2}$ is characteristic in $T_{1} R_{2} A_{0}$.

(ii) $R_{2}$ is characteristic in $T_{1} R_{2}$ and in $T_{1} R_{2} A_{0} T_{3}$.

Proof. The abelian subgroups of maximal order in $R_{0} A_{0}=T_{1} R_{2} A_{0}$ are the groups $B R_{2}$ where $B \leqslant T_{1}$ and $B \cong Z_{4}^{n}$. For if $B_{1}<T_{1} R_{2} A_{0}$ is abelian and $B_{1} \leqslant R_{0}$, then $\left[B_{1}, x\right] \neq 1$ for any $x \in R_{2}-A_{1},\left|B_{1} \cap R_{0}\right| \leqslant 2^{2 n}$ and $\left|B_{1}\right| \leqslant$ $\left|A_{0}\right|^{2 n}<2^{3 n}=\left|B R_{2}\right|$. So $R_{0}=J\left(R_{0} A_{0}\right)$ and $R_{0}$ is characteristic in $R_{0} A_{0}$. Also $R_{2}=Z\left(R_{0}\right)$. Similarly, $R_{0}=J\left(T_{1} R_{2} A_{0} T_{3}\right)$ and we get the result.

Notation. Let $A_{0}=\langle t\rangle$ and $Y=N\left(T_{1} R_{2} A_{0}\right)$. So $Y \leqslant N\left(R_{2}\right)$ by (6.3). Set $\Omega=\left\{\left(A R_{2} / R_{2}\right)^{Y}\right\}$ and let $Y^{*}$ be the induced group of $Y$ on $\Omega$. Set $S_{1}=$ $T_{1} R_{2} A_{0} T_{3}$ and $S_{1} \leqslant S \in \operatorname{Syl}_{2}(G)$ with $S \cap Y \in \operatorname{Syl}_{2}(Y)$.

(6.4) $\Omega \neq\left\{A R_{2} / R_{2}\right\}$.

Proof. Suppose that $\Omega=\left\{A R_{2} / R_{2}\right\}$ so that $A_{0} R_{2} \triangleleft Y$. Then

$$
Y<N\left(T_{1} R_{2} A_{0} \cap C\left(A R_{2}\right)\right)=N\left(T_{1}\right) .
$$

If $R_{2}$ is elementary, then $A_{1}\langle t\rangle=t^{G} \cap R_{2}\langle t\rangle$ and so $S_{1}=S$. We can obtain the structure of $S \cap Y$ in case $R_{2}$ is homocyclic of rank $n$ as follows. First note that $Y$ cannot involve $L_{3}(2)$ in its action on $R_{2} T_{1} / T_{1}$, since using the squaring map $Y$ would then involve $L_{3}(2)$ in its action on $A_{1}$. But then $Y$ involves $L_{3}(2)$ in its action on $T_{1}$ and the argument in (3.14) give a contradiction. Now apply (2.9) to the group $Y / T_{1}$ (setting $U=R_{2} T_{1} / T_{1}$ and $\left.R=A_{0} T_{1} / T_{1}\right)$. We may assume $S \cap Y=U_{1} T_{3} A_{0}$ where $T_{1} R_{2}<U_{1}$ and $U_{1}$ is invariant under $T_{3} A_{0}$ and there is a subgroup $E_{1} \leqslant N\left(U_{1}\right)$ such that $E_{1}$ acts as $D_{1}$ on $T_{1} R_{2}$. So $E=E_{1}^{q-1}$ centralizes $U_{1} / T_{1}$. Also $E_{1}$ is irreducible on $T_{1} / A_{1}$ and $E$ does not centralize $T_{1} / A_{1}$. It follows that $U_{1} / A_{1}=T_{1} / A_{1} \times$ $U_{2} / A_{1}$ for $U_{2} / A_{1}=C_{U_{1} / A_{1}}(E)$. Also $U_{2}=C_{U_{1}}(E)$ and $U_{2} / A_{1} \cong U_{1} / T_{1}$. By (2.4) and the fact that $R_{2}$ is homocyclic of rank $n, U_{2}$ is homocyclic of rank $n$, or $U_{2}$ is isomorphic to a Sylow 2-subgroup of $U_{3}(q)$ or $L_{3}(q)$. As in (6.2) $\left[U_{2}, T_{1}\right]=1$.

If $R_{2}$ is elementary, set $U_{2}=R_{2}$, so that we have the group $U_{2}$ in all cases. 
We next determine $S$. If $U_{2}=R_{2}$ is elementary then we have already noticed that $S=S_{1}=T_{1} R_{2} T_{3} A_{0}$. Suppose $U_{2}$ is nonabelian. Then

$$
N_{S}(S \cap Y) \leqslant N_{S}\left(Z\left(\Omega_{1}(S \cap Y)\right)\right)=N\left(A_{1}\right)
$$

and $T_{1} U_{2} / A_{1}$ is unique of its isomorphic type in $(S \cap Y) / A_{1}$. Consequently, $T_{1} U_{2} \unlhd N_{S}(S \cap Y)$. Fix $s \in N_{S}(S \cap Y)-S \cap Y$. Then $C_{T_{1} U_{2}}\left(t^{s}\right) \cong T_{1}$. If $t^{s} \in T_{1} U_{2} t$, then, as $U_{2}\langle t\rangle \leqslant C\left(T_{1}\right), t^{s} \in U_{2} t$. But

$$
t^{G} \cap U_{2} t=R_{2} t=t^{U_{2}}
$$

so $s \in U_{2} C(t) \leqslant S \leqslant Y$, a contradiction. So $t^{s} \in T_{1} U_{2}\left(T_{3} A_{0}-A_{0}\right)$. $t^{s}$ normalizes $T_{1}$ and $U_{2}$, and $C_{T_{1} U_{2} / A_{1}}(t) \cong C_{T_{1} U_{2} / A_{1}}\left(t^{s}\right)$ has order $q^{3}$. It follows that $t^{s}$ centralizes $U_{2} / A_{1}$ and, hence, $U_{2}\langle t\rangle / A_{1}$. Then for $x \in U_{2}\langle t\rangle$,

$$
\left[x^{2}, t^{s}\right]=\left[x, t^{s}\right]^{x}\left[x, t^{s}\right]=1
$$

so $t^{s}$ centralizes $R_{2}$.

Therefore $R_{2}^{s^{-1}} \leqslant T_{1}$ and is inverted by an element of $T_{1} U_{2} T_{3} A_{0}$. Hence, $A_{0} T_{3}$ is noncyclic and

$$
R_{2}^{s^{-1}} / A_{1}=C_{T_{1} / A_{1}}\left(\Omega_{1}\left(T_{3}\right)\right)
$$

Thus $\left|N_{S}(S \cap Y): S \cap Y\right|=2$. We easily see that $A_{1}$ is characteristic in $N_{S}(S \cap Y)$, and so $T_{1} U_{2}$ is characteristic in $N_{S}(S \cap Y)$. From the action of $N_{S}(S \cap Y)$ on $T_{1} U_{2} / A_{1}$ we now see that $N_{S}(S \cap Y)=S$. Let $\Omega_{1}\left(T_{3}\right)=$ $\langle v\rangle$. Without loss $t^{s} \in t v T_{1} U_{2}$. Since $T_{1} / A_{1}$ is a free $F_{2}\langle t v\rangle$-module we may assume that $t^{s} \in t v U_{2}$. Also $t^{s}$ centralizes $U_{2}\langle t\rangle / A_{1}$, so $t v$ centralizes $U_{2} / A_{1}$ and, hence, $U_{2}\langle t\rangle / A_{1}$. Arguing as in the previous paragraph we obtain $t v \in C\left(R_{2}\right)$. Write $t^{s}=t v u_{2}$ with $u_{2} \in U_{2}$. Then $u_{2} \in C_{U_{2}}\left(R_{2}\right)=R_{2}$. We are assuming that $R_{2}$ is not elementary abelian, so for $t^{s}$ to be an involution we must have $u_{2} \in A_{1}$. Conjugating $t^{s}$ by an element of $T_{1}$ we may assume that $t^{s}=t v$.

Now by (2.6), $T_{1} U_{2} / A_{1}$ is strongly closed in $S / A_{1}$, whence by Goldschmidt [11], the action of $E_{1}$, and the knowledge of the appropriate Schur multipliers, we conclude that $\bar{T}_{1} \bar{U}_{2} \unlhd \overline{N\left(A_{1}\right)}$ (where bars refer to images in $N\left(A_{1}\right)$ $\left./ O\left(N\left(A_{1}\right)\right)\right)$. Let $x \in S-Y$ and suppose $x \sim t . T_{1} U_{2} / A_{1}$ is an $\mathrm{F}_{2}\langle x\rangle-$ module, so if $C / A_{1}=C(x) \cap T_{1} U_{2} / A_{1}$, then $|C| \geqslant q^{3}$. As $x$ centralizes $C / A_{1},\left|C_{C}(x)\right| \geqslant q^{2}$. Any subgroup $X$ of $T_{1}$ of order $q^{2}$ contains $A_{1}$. For otherwise, with $H$ a subgroup of $A_{1}$ of index 2 containing $X \cap A_{1}$, and bars indicating images modulo $H, \bar{T}_{1}$ is extraspecial of order $2 q^{2}$ and $\bar{X}$ is a subgroup of order $>q$ meeting $Z\left(\bar{T}_{1}\right)$ trivially. This is impossible. Choose $g$ with $x^{g}=t$ and $C_{S}(x)^{g} \leqslant S$. By the above we have $t_{1} \in T_{1}$ satisfying

$$
t^{t^{-1} x t_{1}}=t^{x t_{1}} \in t v A_{1} \text {, }
$$

so we may assume that $\left[t, t^{x}\right]=1$. Considering the action of $x$ on $C_{G}(t) \cap$ $C_{G}\left(t^{x}\right)$ we have $\left|C_{A_{1}}(x)\right|=q$ or $\sqrt{q}$. Since $|C| \geqslant q^{3}$ and all involutions in 
$A_{1} x$ are in $C_{A_{1}}(x) x$, we conclude that $\left|C_{C}(x)\right| \geqslant q^{2}$ or $q^{2} \sqrt{q}$, respectively, and in either case $\Phi\left(C_{C}(x)\right) \leqslant C_{A_{1}}(x)$.

Set $J=\langle x\rangle \times\left\langle t t^{x}\right\rangle C_{C}(x)$. Then $J^{g} \leqslant C_{S}(t)=T_{1} T_{3} A_{0}$. As $\langle x\rangle \times C_{C}(x)$ has class at most $2,\left(\langle x\rangle \times C_{C}(x)\right)^{g}\left\langle T_{1}\langle t, v\rangle\right.$. Suppose $C_{A_{1}}(x)=A_{1}$. Then for $r_{2} \in R_{2}-A_{1}, x$ centralizes the involution $r_{2} r_{2}^{x}$, and so $C_{C}(x)$ contains an elementary abelian group of order $q^{2}$. This implies that $\left(\langle x\rangle \times C_{C}(x)\right)^{g} \leqslant$ $T_{1}\langle t, v\rangle$ contains an elementary subgroup of order $2 q^{2}$, a contradiction. Therefore $\left|C_{A_{1}}(x)\right|=\sqrt{q}$. Then $y \in C_{C}(x)$ implies that

$$
\left|\left[C_{C}(x), y\right]\right| \leqslant \sqrt{q} \text { and }\left|C_{C}(\langle x, y\rangle)\right| \geqslant q^{2} .
$$

Therefore

$$
y^{g} \leqslant T_{1}\langle t\rangle \text { and }\left(C_{C}(x)\langle x\rangle\right)^{8} \leqslant T_{1}\langle t\rangle \text {. }
$$

As $C_{C}(x)\langle x\rangle$ has order at least $2 q^{2} \sqrt{q}$, we must have $A_{1} \leqslant \Phi\left(C_{C}(x)\right)$, whereas $\Phi\left(C_{C}(x)\right) \leqslant C_{A_{1}}(x)$ has order at most $\sqrt{q}$. This is a contradiction. We have now shown that $t^{G} \cap S \subseteq S \cap Y$.

Now $t \not \partial v$ as $v$ centralizes an elementary abelian subgroup of $T_{1} U_{2}$ of order $q^{2}$. Hence $t^{G} \cap\langle v\rangle T_{1} U_{2}=\varnothing$. Next suppose that $v \sim x$ with $x$ extremal and $x \in T_{1} U_{2}$ or $x \in S-Y$. Choose $g \in G$ with $v^{g}=x, C_{S}(v)^{g} \leqslant$ $C_{S}(x)$. Then $t^{g} \sim_{S} t$, because we have shown that $t^{G} \cap S \leqslant T_{1} U_{2}\langle v\rangle t$ and $S$ controls fusion in $t^{G} \cap T_{1} U_{2}\langle v\rangle t$. So $t^{g}=t$, without loss. Therefore $g \in$ $N\left(M_{1}\right)$ and $v^{g} \in C(t)$. Then $v^{g} \in S \cap Y$, and, $x \notin T_{1} U_{2}$. This is a contradiction. Therefore $v$ is extremal.

We have $C_{S}(v)=F T_{3}\langle t\rangle\left\langle s_{1}\right\rangle$, where $F=C_{T_{1} U_{2}}(v)$ is elementary of order $q^{2}$, and $s_{1}=1$ or $s_{1} \in S-Y$. By the above $v$ is extremal in $S$ so that $C_{S}(v) \in \operatorname{Syl}_{2}\left(C_{G}(v)\right)$. Set $S_{0}=C_{S}(v)$ and $I=C_{G}(v)$. We study the strong closure, $J$, of $F\langle v\rangle$ in $S_{0}$ (with respect to $I$ ). We have already seen that $t^{G} \cap F\langle v\rangle=\varnothing$. Since $t^{G} \cap F t=A_{1} t=t^{F}$, and since no element of $F t-$ $A_{1}\langle t\rangle$ is an involution, we conclude that no element of $F t$ is conjugate to an element of $F^{\#}$. Similarly, $t \sim t v$ and $t^{G} \cap F t v=A_{1} t v=(t v)^{F}$ imply that no element of $F t v$ is conjugate to an element of $F$. Let $a \in(F\langle v\rangle)^{\#}$ and suppose $a^{I} \cap S_{0} \& F\langle v\rangle$. Say $b \in\left(a^{I} \cap S_{0}\right)-F\langle v\rangle$.

No element of $F T_{3}-F\langle v\rangle$ is an involution, so, by the above, $b \in S_{0}-$ $F T_{3}\langle t\rangle$. If $F\langle v\rangle$ is not weakly closed in $S_{0}$ (with respect to $I$ ), then for some $g \in I, F\langle v\rangle \neq(F\langle v\rangle)^{g} \leqslant S_{0}$, so $F\langle v\rangle \cap(F\langle v\rangle)^{g}$ is maximal in $F\langle v\rangle$, and we may assume that $b$ centralizes a maximal subgroup of $F\langle v\rangle$. If $F\langle v\rangle$ is weakly closed in $S_{0}$, then this also holds, as is seen from (2.6). We claim that $b$ must centralize $F$. As $b$ normalizes $T_{1} U_{2}, b$ normalizes $C_{T_{1} U_{2} / A_{1}}(F)=\hat{F} / A_{1}$, where $\hat{F}=T_{11} R_{2}, T_{11} \leqslant T_{1}$, and $T_{11}$ is homocyclic of rank $n$. In particular, $\hat{F}$ is abelian, $\Omega_{1}(\hat{F})=F$, and $\mho_{1}(\hat{F})=A_{1}$. Consider the action of $b$ on $\hat{F} / A_{1}$. Since $t^{b} \in t v F, t^{b} \in t v A_{1}$, and so $T_{1} \cap T_{1}^{b} \leqslant C_{T_{1}}(t v)=A_{1}$. Consequently, $\hat{F} / A_{1}=T_{11} / A_{1} \times T_{11}^{b} / A_{1}$ and $\hat{F} / A_{1}$ is a free $\mathrm{F}_{2}\langle b\rangle$-module. If $b$ centralizes 
$A_{1}$, then $g \in \hat{F}$ implies that $g g^{b}$ is an involution centralized by $b$, and it follows that $b$ must centralize $F$. If $b$ does not centralize $A_{1}$, then $[F, b]=$ $\left[A_{1}, b\right]$ has order 2 and $F / A_{1}=C(b) \cap \hat{F} / A_{1}$. In this case there is an element $g \in T_{11}$ with $g g^{b}$ of order 4 (just choose $g$ so that $\left(g^{2}\right)^{b} \neq g^{2}$ ). But then $g g^{b} A_{1} \notin F / A_{1}$ although $b$ centralizes $g g^{b} A_{1}$. This is a contradiction, so the claim is proved.

In view of the above claim we conclude that either $J=F\langle v\rangle$ or $J=$ $F\langle v\rangle\langle b\rangle=C_{S_{0}}(F\langle v\rangle)$. Clearly $J$ is weakly closed in $S_{0}$, and using (2.6) we have $J$ strongly closed in $S_{0}$. We can then apply Goldschmidt [11] and conclude that $E(I / O(I)) \cong L_{2}\left(q^{2}\right)$ or $L_{2}(q) \times L_{2}(q)$. Let $H$ be a 2-complement in $N(F) \cap C(v)^{(\infty)}$. We consider the group $N=N_{0} H$, where $N_{0}=$ $N_{G}(F\langle v\rangle) \cap C_{G}(F)$. Then $N_{0} \unlhd N$ and, since $v^{G} \cap F=\varnothing, N$ acts on $F v$. Also $P=N_{0} \cap T_{1} U_{2}$ has order $q^{4}$ (check this directly using the fact that $\left.\left[T_{1}, U_{2}\right]=1\right)$. As $C_{P}(v)=F, P$ is transitive on $F v$. Consequently, $N_{0}$ and $N$ are each transitive on $F v$.

In $N_{0}$ the stabilizer of $v$ must centralize $F\langle v\rangle$. So let $N_{1}=C_{N}(F\langle v\rangle)$. Then $N=N_{1} P H$ and $H$ acts on $P N_{1} / N_{1}$. Since $C_{S}(v) \in \operatorname{Syl}_{2}\left(C_{G}(v)\right)$, and since $C_{S}(v)$ normalizes $N_{0}$, we conclude that $C_{S}(v) \cap N_{1} \in \operatorname{Syl}_{2}\left(N_{1}\right)$. Consequently, $F\langle v\rangle$ has index at most 2 in a Sylow 2-subgroup of $N_{1}$, and $\hat{S}=S \cap N_{0} \in \operatorname{Syl}_{2}\left(N_{0}\right)$. Now $N_{N}(\hat{S})$ covers $N / N_{0}$ and we observe that a 2-complement of $N_{N}(\hat{S})$ induces an abelian group on $\hat{S}$. For let $H_{1}$ be a 2-complement in $N_{N}(\hat{S})$. Then $H_{1} / C_{H_{1}}(F) \cong H / C_{H}(F)$ is abelian. Also $C_{H_{1}}(F)\left\langle N_{1}\right.$, so $\left[P, C_{H_{1}}(F)\right]\left\langle F\langle v\rangle\langle g\rangle\right.$, where $g^{2} \in F\langle v\rangle$ and $g \in$ $N(F\langle v\rangle)$. Since $C_{H_{1}}(F)$ centralizes $F$ and normalizes $F\langle v\rangle,\left[P, C_{H_{1}}(F)\right]=1$, proving the claim. Since $E_{1}^{q+1} \leqslant F H$ and $\left[\hat{S}, E_{1}^{q+1}\right]=P, N_{N}(\hat{S}) \leqslant N_{N}(P)$. Therefore $N_{N}(\hat{S}) \leqslant N\left(P^{\prime}\right)=N\left(A_{1}\right)$. But $H\langle t\rangle$ acts irreducibly on $F$ and $N_{N}(\hat{S})$ induces $H / C_{H}(F)$ on $F$. This is a contradiction.

We are left with the possibility that $U_{2}$ is homocyclic of rank $n$. If $U_{2}=R_{2}$, then $S=S_{1}=T_{1} R_{2} T_{3} A_{0}$, so suppose $U_{2}>R_{2}$. Checking centralizers we see that

$$
R_{2} t \subseteq t^{N(S \cap Y)} \subseteq U_{2} t
$$

So

$$
N(S \cap Y) \leqslant N\left(C_{S \cap Y}\left(t^{S \cap Y}\right)\right) \leqslant N\left(T_{1}\right) \text { or } N\left(T_{1} \Omega_{1}\left(T_{3}\right)\right),
$$

and as $T_{1}$ is characteristic in $T_{1} \Omega_{1}\left(T_{3}\right)$, we have $N(S \cap Y) \leqslant N\left(T_{1}\right)$. Consider the group $I=C_{G}\left(T_{1}\right) E_{1}$. We have $(S \cap Y) \cap I=U_{2}\langle t\rangle$. We apply (2.9) to $I$. Even though we may have $q=4$, the arguments used in the proof of (2.9) all carry through in this case as well. We conclude that there is a homocyclic group $\hat{U}_{2} \geqslant U_{2}$ such that $\hat{U}_{2}$ has rank $n$ and $\hat{U}_{2}\langle t\rangle \in$ $\operatorname{Syl}_{2}\left(C\left(T_{1}\right)\right)$. We may assume that $T_{3} \leqslant N\left(\hat{U}_{2}\right)$. Letting $S_{2}=T_{1} \hat{U}_{2} T_{3} A_{0}$, we may assume $S_{2} \leqslant S$. Choose $g \in N_{S}\left(S_{2}\right)-S_{2}$. Then $g \in N\left(Z\left(\Omega_{1}\left(S_{2}\right)\right)\right)=$ 
$N\left(A_{1}\right)$, and since $T_{1} \hat{U}_{2} A_{0} / A_{1}$ is the unique group of its isomorphism type in $S_{2} / A_{1}$, we have $g \in N\left(T_{1} \hat{U}_{2} A_{0}\right)$. Checking centralizers we have $t^{g} \in \hat{U}_{2} t$. So $g$ normalizes

$$
C_{T_{1} \hat{U}_{2} A_{0}}\left(\left\langle t^{N_{S}\left(S_{2}\right)}\right\rangle\right)=T_{1} .
$$

Now consider $N\left(T_{1}\right) / T_{1}$. We argue as in (3.14) that $N\left(T_{1}\right)$ does not involve $L_{3}(2)$. As $g$ normalizes $T_{1} \hat{U}_{2}\langle t\rangle / T_{1}$ we have $\left\langle E_{1}, g\right\rangle$ inducing a 2 -transitive group on $T_{1} \hat{U}_{2}\langle t\rangle / T_{1} \mho_{1}\left(\hat{U}_{2}\right)$. Using the arguments of (2.7) together with an application of the 3-subgroups theorem we conclude that there is a 2-group $\tilde{U}_{2}>\hat{U}_{2}$ with $\left[\tilde{U}_{2}, t\right]=\hat{U}_{2}$ and $\tilde{U}_{2} \leqslant C\left(T_{1}\right)$. However, $\hat{U}_{2}\langle t\rangle \in \operatorname{Syl}_{2}\left(C\left(T_{1}\right)\right)$. This is a contradiction. We conclude that $S=T_{1} \hat{U}_{2} T_{3} A_{0}$. We now have $S$ in all cases. If $R_{2}=U_{2}$, let $\hat{U}_{2}=U_{2}$. Then $S=T_{1} \hat{U}_{2} T_{3} A_{0}$.

If $T_{3} A_{0}$ is cyclic, then we have a contradiction as follows. It is easy to check centralizers to get $t^{G} \cap T_{1} \hat{U}_{2}=\varnothing$. So by the Thompson transfer lemma and the action of $\left.D_{1}, T_{1} \hat{U}_{2} \in \operatorname{Syl}_{2}\left(\left\langle T_{1} \hat{U}_{2}\right)^{G}\right\rangle\right)$, against (3.3). We may assume that $T_{3} A_{0}=T_{3} \times A_{0}>A_{0}$ and let $\langle v\rangle=\Omega_{1}\left(T_{3}\right)$.

Let $X=N_{G}\left(A_{1}\right)$ and let bars denote images in $X / A_{1}=\bar{X}$. Then using (2.6) we have $T_{1} \hat{U}_{2}$ strongly closed in $\bar{S}$ unless possibly $\hat{U}_{2}=R_{2}$, in which case $\overline{T_{1} \hat{U}_{2} A_{0}}$ is strongly closed in $\bar{S}$. By Goldschmidt [11] $\left(\bar{T}_{3} \times \bar{A}_{0}\right) \cap\left\langle\left(\bar{T}_{1} \overline{\hat{U}}_{2}\right)_{\bar{X}}\right\rangle$ $=1$. From here we check centralizers of elements of $T_{3} \times A_{0}$ acting on $\bar{T}_{1} \hat{U}_{2}$ and use the fact that $\bar{S} / \overline{T_{1} \hat{U}_{2}}$ is abelian to see that

$$
t^{X} \cap T_{1} \hat{U}_{2} v=t^{X} \cap T_{1} \hat{U}_{2} t v=\varnothing .
$$

In particular, $t \not \chi v$ and $t \not x t v$ in $X$. Using the action of $E_{1}$ on $\bar{T}_{1} \overline{\hat{U}}_{2}$ and information on multipliers, we conclude that $T_{1} \hat{U}_{2} O(\bar{X}) \unlhd \bar{X}$.

If $t^{g}=v$ or $t v$, then for some Sylow 2-subgroups, $H$, of $M_{1}^{g}, A_{1}=\Omega_{1}(H)$ and we may suppose $g \in N\left(A_{1}\right)$. But we have just seen this to be impossible. So $t \not v$ and $t \not t v$. Consequently, if we set $K=C_{G}(v) /\langle v\rangle$, then $M_{2}=$ $C_{M_{1}}(v) /\langle v\rangle$ is *-standard in $K$ and $N_{K}\left(M_{2}\right) \cap C\left(\tilde{M}_{2}\right)$ contains $\langle t, v\rangle /\langle v\rangle$ as a Sylow 2-subgroup. By induction we have the structure of $K / O(K)$. Similarly for $C_{G}(t v) /\langle t v\rangle$.

We claim that $t^{G} \cap T_{1} \hat{U}_{2}\langle v\rangle=\varnothing$. For suppose $t^{g} \in T_{1} \hat{U}_{2}\langle v\rangle$. Then $A_{1} \leqslant$ $C\left(t^{8}\right)$ and we first show that $A_{1} \leqslant M_{1}^{g}$. If $a \in A_{1}-M_{1}^{g}\left\langle t^{8}\right\rangle$, then since all involutions in $M_{1}^{g} a$ are conjugate we have $a \sim v$ or $t v$. But then $C_{G}(v)$ or $C_{G}(t v)$ contains a conjugate of $T_{1} \hat{U}_{2}\langle t, v\rangle$, contradicting the above paragraph. Therefore $A_{1} \leqslant M_{1}^{g}\left\langle t^{g}\right\rangle$. All involutions in $M_{1}^{g} t^{g}$ are fused to $t^{g}$, so $A_{1} \leqslant M_{1}^{g}$. So we may assume that $g \in X=N_{G}\left(A_{1}\right)$, whereas we have already shown that $t^{X} \cap T_{1} \hat{U}_{2}\langle v\rangle=\varnothing$. So the claim holds and, similarly, $t^{G} \cap T_{1} \hat{U}_{2}\langle t v\rangle=\varnothing$.

Apply the Thompson transfer lemma to $S$, with $S_{0}=T_{1} \hat{U}_{2} T_{3}$. By the above, $t \notin G^{\prime}$, although from the structure of $N\left(T_{1} \hat{U}_{2}\right)$ we have $T_{1} \hat{U}_{2} \leqslant G$. So $S \cap G^{\prime}=T_{1} \hat{U}_{2}\langle l\rangle$ where $\Omega_{1}(\langle l\rangle)=\langle v\rangle$, $\langle t v\rangle$, or 1 . Any involution in 
$T_{1} \hat{U}_{2}$ is centralized by an abelian subgroup of order $q^{3}$. But from the known structure of $C_{G}(t)$ and $C_{G}(t v)$, we conclude that $v^{G} \cap T_{1} \hat{U}_{2}=(t v)^{G} \cap T_{1} \hat{U}_{2}$ $=\varnothing$. Consequently, we may apply the Thompson transfer theorem once again and obtain a normal subgroup of $G$ with $T_{1} \hat{U}_{2}$ as Sylow 2-subgroup. This contradicts (3.3), completing the proof of (6.4).

(6.5) $|\Omega|=q^{2}, Y^{*}$ is transitive on $\Omega$, and $Y^{*}$ contains a regular normal subgroup.

Proof. As $A_{0}^{G} \cap T_{1} R_{2}=\varnothing$ (check centralizers), $\Omega \subseteq t T_{1} R_{2} / R_{2}$ and $|\Omega| \leqslant$ $q^{2}$. Using the action of $D_{1}$ we see that if $\Omega$ is not of order $q^{2}$, then $|\Omega|=1+\frac{1}{3}\left(q^{2}-1\right)$ or $1+\frac{2}{3}\left(q^{2}-1\right)$. In the first case $Y^{*}$ satisfies the hypotheses, but not the conclusion, of Theorem 1.1 of Hering, Kantor and Seitz [16]. In the second case $|\Omega|$ is odd, so $Y^{*}$ has cyclic Sylow 2-subgroup and is solvable. By order considerations $Y^{*}$ is primitive. Also $D_{1}^{*}$ is semiregular on $\Omega-\left\{A R_{2} / R_{2}\right\}$. So if $N^{*}$ is a minimal normal subgroup of $Y^{*}, N^{*}$ is semiregular on $\Omega$ and $N^{*} D_{1}^{*}$ is a Frobenius group. But then $D_{1}^{*}$ cannot act fixed-point-freely on $T_{1} R_{2} / R_{2}$. So $|\Omega|=q^{2}$ and, by definition of $\Omega, Y^{*}$ is transitive on $\Omega$.

It remains to show that $Y^{*}$ contains a regular normal subgroup. In the action on $\Omega, \Omega_{1}\left(T_{3}\right)$ fixes all (if $T_{3}=1$ ) or exactly $q$ points of $\Omega$, so if $T_{3}^{*} \neq 1$, then $C_{Y^{*}}\left(\Omega_{1}\left(T_{3}\right)^{*}\right)$ has Sylow 2-subgroups of order dividing $q\left|T_{3}\right|$. If $n$ is even then $3 \nmid q+1, Y^{*}$ is 2-transitive and Theorem (1.1) of [16] gives the result. So suppose $n$ is odd. Then $\left|T_{3}\right|=1$ or 2 . Consider $Y_{0}=C_{Y}\left(A_{1}\right) \unlhd Y$. Then $Y_{0}^{*}$ has orbits of equal length on $\Omega$ and the stabilizer in $Y_{0}^{*}$ of $A R_{2} / R_{2}$ is $D^{*} \Omega_{1}\left(T_{3}\right)^{*}$. So $\left|Y_{0}^{*}\right|=(q+1) 2^{a}$ or $\frac{1}{3}(q+1) 2^{a}$ for some integer $a \geqslant 2$. Choose $N$ minimal normal in $Y^{*}$ with $N \leqslant Y_{0}$. If $D^{*} \cap N=1$, then $N$ is a 2-group and is consequently a regular normal subgroup. So suppose $D^{*} \cap N$ $\neq 1$. But $\left|N: C_{N}\left(D^{*} \cap N\right)\right|$ is a power of 2 , so we obtain a contradiction from Burnside's Theorem.

Notation. Let $Y_{1}$ be the kernel of $Y$ on $\Omega$ and let $U$ be a Sylow 2-subgroup of the preimage of the regular normal subgroup of $Y^{*}$. We may assume $R_{2}\langle t\rangle \leqslant U \cap Y_{1}$, that $T_{1} R_{2} \leqslant U$, and that there is a subgroup $E_{1}$ of odd order such that $T_{3}$ normalizes $E_{1}, E_{1}$ normalizes $U$, and $E_{1}$ induces $D_{1}$ on $T_{1} R_{2}$. Then $U=U_{1}\langle t\rangle$, where $U_{1}=\left[U, E_{1}\right]$. Let $\hat{R}_{2}=U_{1} \cap Y_{1}$. Standard arguments imply $R_{2}=\hat{R}_{2}$ or $\hat{R}_{2} / R_{2} \cong A_{1}$ as $E_{1}$-modules.

(6.6) Let $U_{0}=\left[U_{1}, E\right]$, where $E=E_{1}^{q-1}$.

(i) $U_{0}$ covers $U_{1} / \hat{R}_{2}$.

(ii) $U_{1} / \hat{R}_{2}$ is elementary abelian.

(iii) $\left[U_{0}, \hat{R}_{2}\right]=1$.

Proof. (i) is clear as $U_{1} / T_{1} \hat{R}_{2}$ and $T_{1} \hat{R}_{2} / \hat{R}_{2}$ are equivalent $E_{1}$-modules. 
Also, by (2.2), $U_{1} / \hat{R}_{2}$ is abelian. As the normal closure of $E$ in $Y$ centralizes $R_{2}, U_{0}$ is in $C\left(\hat{R}_{2}\right)$, proving (iii).

So $U_{0}$ has class 2 and for $x, y \in U_{0},\left[x^{2}, y^{2}\right]=\left[x^{4}, y\right] \in\left[\hat{R}_{2}, y\right]=1$. If $U_{1} / \hat{R}_{2}$ is not elementary, this would imply $T_{1}$ is abelian, which is absurd. This proves (ii).

(6.7) (i) $R_{2}$ is elementary abelian, $R_{2}=\hat{R}_{2}$, and $U_{1}^{\prime}=R_{2}$.

(ii) $R_{2}$ and $U_{1}$ are characteristic in $U_{1} T_{3} A_{0}=\hat{U}$.

Proof. Suppose that $R_{2}$ is homocyclic of rank $n$. As $U_{0}$ has class 2 and $\hat{R}_{2} \cap U_{0}<Z\left(U_{0}\right), x, y$ in $U_{0}$ implies that $1=\left[x^{2}, y\right]=[x, y]^{2}$. So $\left[U_{0}, U_{0}\right]<$ $A_{1}$ and for $x \in U_{0},\left|C_{U_{1}}(x)\right| \geqslant q^{5}$. Choose $x \in T_{1}-A_{1}$. Then $t$ normalizes $C_{U_{1}}(x)$ and $\left|C_{T_{1}}(x)\right|=q^{2}$. Consequently, $U_{1}=T_{1} C_{U_{0}}(x)$. As $\left[U_{1}, t\right] R_{2}=$ $T_{1} R_{2}$, we obtain a contradiction by looking at $\left[t, C_{U_{1}}(x)\right]$. Namely, $C_{U_{1}}(x)$ covers $U_{1} / T_{1} R_{2}$, so

$$
T_{1} R_{2}=\left[t, U_{1}\right] R_{2}=\left[t, C_{U_{1}}(x)\right] R_{2} .
$$

On the other hand, $\left[t, C_{U_{1}}(x)\right] \leqslant C_{T_{1} R_{2}}(x)$, which does not cover $T_{1} R_{2} / R_{2}$. So $R_{2}$ is elementary and $R_{2}=\hat{R}_{2}$. Also the above argument shows that $U_{1}^{\prime}=R_{2}$.

By (6.6) $R_{2} \leqslant Z\left(U_{1}\right)$. Also $\left[U_{1}, t\right] R_{2}=T_{1} R_{2}$ and $\hat{U}^{\prime}\left\langle U_{1}\right.$, so $\left.C_{\hat{U}}\left(\hat{U}^{\prime}\right)\right\rangle$ $R_{2}$, is $t$-invariant and intersects $T_{1}$ in $A_{1}$. This forces $C_{\hat{U}}\left(\hat{U}^{\prime}\right)=R_{2}$, so $R_{2}$ is characteristic in $\hat{U}$. In $\hat{U} / R_{2}, U / R_{2}$ is unique of its isonorphism type, proving (ii).

(6.8) $t^{G} \cap U_{1}\langle t\rangle=t^{U_{1}}=U_{1} t$.

(ii) $T_{3} A_{0}=T_{3} \times A_{0}>A_{0}$.

Proof. Both $U_{1} / R_{2}$ and $R_{2}$ are free $\mathrm{F}_{2}\langle t\rangle$-modules, proving (i) (no conjugate of $t$ centralizes $R_{2}$, so $t^{G} \cap U_{1}=\varnothing$ ). If $T_{3} A_{0}$ is cyclic, then from (6.7)(ii) and (i) we have $S=U_{1} T_{3} A_{0}$. However, we can then transfer out $T_{3} A_{0}$, contradicting (3.3). So $T_{3} A_{0}$ is not cyclic and (ii) holds.

(6.9) $S>U_{1} T_{3} A_{0}$.

PRoof. Suppose that $S=U_{1}\left(T_{3} \times A_{0}\right)$. First assume that $C_{S}\left(R_{2}\right)>U_{1}$. Then $C_{S}\left(R_{2}\right)=U_{1}\langle k\rangle$, where $\langle k\rangle$ or $\langle k t\rangle$ is $\Omega_{1}\left(T_{3}\right)$. By (3.5)(a) and transfer, $G=O^{2}(G) A_{0}$ with $O^{2}(G) \cap A_{0}=1$. Clearly $U_{1} \leqslant O^{2}(G)$, so $U_{1}\langle l\rangle \in$ $\operatorname{Syl}_{2}\left(O^{2}(G)\right.$ ) with $\Omega_{1}(\langle l\rangle)=\left\langle k^{\prime}\right\rangle$ and $k^{\prime}=k$ or $k t$. If $\left(k^{\prime}\right)^{O^{2}(G)} \cap U_{1}=\varnothing$, then we have a contradiction by transfer. So assume $\left(k^{\prime}\right)^{G} \cap U_{1} \neq \varnothing$. Involutions in $U_{1}$ have centralizers of order at least $q^{4}$ (as $U_{1}^{\prime}=R_{2}$ ). Since $U_{1} / R_{2}$ is a free $\mathrm{F}_{2}\left\langle k^{\prime}\right\rangle$-module, each involution in $U_{1} k^{\prime}$ is conjugate to one in $R_{2} k^{\prime}$. Now $C_{U_{1}}\left(k^{\prime}\right)$ does not cover $C\left(k^{\prime}\right) \cap U_{1} / R_{2}$, since $C_{T_{1}}\left(k^{\prime}\right)=A_{1}$. Consequently, elements of $k^{\prime G} \cap U_{1}\left\langle k^{\prime}\right\rangle$ that are extremal in $U_{1}\langle l\rangle$ are all in $U_{1}$. Choosing a Sylow 2-subgroup of $C\left(k^{\prime}\right)$ and extending to a Sylow 2-subgroup of $G$, we see that there is a conjugate $U_{1}^{g}$ of $U_{1}$ such that $k^{\prime} \in U_{1}^{g}$ 
and $t \in N\left(U_{1}^{g}\right)$. Since $t \notin U_{1}^{g}$, both $U_{1}^{g} / R_{2}^{g}$ and $R_{2}^{g}$ are free $\mathbf{F}_{2}\langle t\rangle$-modules. It follows that $\left|C(t) \cap U_{1}^{g}\right|=q^{3}$. Modulo $O(M), C(t) \cap U_{1}^{g}\langle t\rangle=T_{1}^{*}\langle t, v\rangle$, where $T_{1}^{*} \leqslant U_{1}^{g}$ and has index 2 in $T_{1}$. But $\left[k, T_{1}\right]$ is homocyclic of exponent 4, whereas $\left[k, U_{1}^{g}\right] \leqslant R_{2}^{g}$. This is impossible.

We now have $C_{S}\left(R_{2}\right)=U_{1}$. Then both $U_{1} / R_{2}$ and $R_{2}$ are free modules for $\langle t\rangle,\langle v\rangle$, and $\langle t v\rangle$, so we conclude that each involution in $S-U_{1}$ is conjugate in $U_{1}$ to one of $t, v, t v$. The arguments of the previous paragraph show that $v^{G} \cap U_{1}=(t v)^{G} \cap U_{1}=\varnothing$, and we know that $t^{G} \cap U_{1}=\varnothing$, as $t$ cannot centralize a conjugate of $R_{2}$. Suppose $t^{G} \cap U_{1}\langle v\rangle=\varnothing$. Then apply the Thompson transfer theorem twice to conclude that $U_{1} \in \operatorname{Syl}_{2}\left(\left\langle U_{1}^{G}\right\rangle\right)$, which contradicts (3.3). So assume that $t^{8} \in U_{1}\langle v\rangle$ for some $g \in G$. By the above we may assume that $t^{g}=v$. Also we may assume $g$ normalizes $C_{M_{1}}(v)^{(\infty)}$ and $A_{1}$. So $\left(A_{1}\langle t\rangle\right)^{g}=A_{1}\langle v\rangle$. A Sylow 2-subgroup of $N_{G}\left(A_{1}\langle v\rangle\right)$ is a conjugate of $T_{1} R_{2} T_{3}\langle t\rangle$. Note that $R_{2} \leqslant N\left(A_{1}\langle v\rangle\right)$. We claim that $R_{2}$ is strongly closed in $T_{1} R_{2} T_{3}\langle t\rangle$ with respect to $G$. Suppose $r \in R_{2}$ and $r^{x} \in$ $T_{1} R_{2} T_{3} A_{0}-R_{2}$. Then $r^{x} \notin T_{1} R_{2}$, as $\Omega_{1}\left(T_{1} R_{2}\right)=R_{2}$. So $\left[t, r^{x}\right] \in T_{1}-A_{1}$. However, $\left\langle t, r^{x}\right\rangle$ is dihedral, so $t$ inverts $\left[t, r^{x}\right]$. This is impossible. By the above we have now established the claim. So we may take $g \in N\left(R_{2}\right)$. Now use (2.6) and Goldschmidt [11] to conclude that $U_{1} O\left(N_{G}\left(R_{2}\right)\right) \unlhd N_{G}\left(R_{2}\right)$. Consequently, we may assume $g \in N\left(U_{1}\right)$.

It now follows that $g$ can be chosen as a 3-element in $N(S)$ and $t \sim v \sim$ tv. In particular, $T_{3}=\langle v\rangle$. Consider the group $N=N_{G}\left(U_{1}\right)$ and let bars denote images in $N / C_{N}\left(U_{1} / R_{2}\right)$. The group $C_{N}\left(R_{2}\right)$ is 2-closed and $\bar{E} \leqslant \overline{C_{N}\left(R_{2}\right)}$. Also, since $g$ normalizes $A_{1}$ and $C_{M_{1}}(v)^{(\infty)}$, we may assume $\bar{g}$ normalizes $\bar{E}_{1}^{q+1}$. Say $\bar{H}$ is minimal normal in $\bar{N}$ with $H \leqslant C_{N}\left(R_{2}\right) . \bar{H}$ is an elementary $l$-group for some prime $l$. Then

$$
\bar{H}=C_{\bar{H}}(\bar{t}) C_{\bar{H}}(\bar{v}) C_{\bar{H}}(\overline{t v}) .
$$

But $C_{\bar{H}}(\bar{t}) \leqslant \bar{E}$ and $C_{\bar{H}}(\bar{t}) \cap C_{\bar{H}}(\overline{t v})=1$. So $\bar{H}$ has order $l^{3}$. As $\bar{E}_{1}^{q+1}$ centralizes $C_{\bar{H}}(\bar{t})$, we use the action of $\bar{g}$ to see that $\bar{E}_{1}^{q+1} \bar{H}=\bar{E}_{1}^{q+1} \times \bar{H}=$ $B$. However, $U_{1} / R_{2}$ has at most $2 B$-composition factors, as $\bar{E}_{1}^{q+1} \times C_{\bar{H}}(\bar{t})$ is irreducible on $T_{1} R_{2} / R_{2}$ and on $U_{1} / T_{1} R_{2}$. This implies $B$ has rank at most 2, and we have a contradiction. This completes the proof of (6.9).

Let $N_{1}=N_{G}\left(U_{1}\right)$ and let bars denote images in $N_{1}$ modulo $C_{N_{1}}\left(U_{1} / R_{2}\right)$. Write $\langle v\rangle=\Omega_{1}\left(T_{3}\right)$. There is an element $s \in N_{s}\left(U_{1} T_{3} A_{0}\right)$ such that $t^{s} \notin U_{1} t$. Now $s$ acts on $T_{3} A_{0} U_{1} / U_{1} \cong T_{3} \times A_{0}$. So if $\left|T_{3}\right|>2$ we have $t^{s} \in U_{1} t v$. If $\left|T_{3}\right|=2$ we rechoose $T_{3}$, if necessary, so that in all cases $t^{s} \in U_{1} t v$. Since each of $R_{2}$ and $U_{1} / R_{2}$ is a free $F_{2}\left(\left\langle t^{s}\right\rangle\right)$-module, we may assume $t^{s}=t v$. Then $s$ normalizes $\bar{N}=C_{\bar{N}_{1}} \overline{\left\langle\left\langle t, t^{s}\right\rangle\right)}$. So $s$ normalizes $\bar{E}_{00}$, where $E_{00}=E_{1}^{q+1}$. Also $s$ normalizes $O\left(\bar{N}_{1}\right) \geqslant \bar{E}$.

Now $\left[\bar{E}_{00}, \bar{E}\right]=\left[\bar{E}_{00}, \bar{E}^{\bar{s}}\right]=1$, so that $W=\left\langle\bar{E}_{00}, \bar{E}, \bar{E}^{\bar{s}}\right\rangle$ centralizes $\bar{E}_{00}$. It 
follows that $V=U_{1} / R_{2}$ may be regarded as an $\mathbf{F}_{q}$-module for $W$ and that $V$ is either irreducible under the action of $W$ or the sum of two irreducibles. That is, $V$ is the sum of irreducible submodules of dimension 1 or 2. Passing to splitting fields and noting that $W$ has odd order, we see that each of the submodules splits into linear factors. Consequently, $W$ is abelian.

Since $t$ centralizes $\bar{E}$ and $t^{s}$ inverts $\bar{E}$, we know that $\bar{E} \bar{E}^{s}=\bar{E} \times \bar{E}^{s}$. From here we see that $V$ splits into a sum $V=V_{1} \oplus V_{2}$ of inequivalent irreducible modules for $\bar{E}_{1} \bar{E}_{1}^{s}=\bar{E}_{00} \times \bar{E} \times \bar{E}^{s}=W$.

This decomposition of $V$ gives further information about $U_{1}$ as follows. As $\bar{E}_{1}$ is fixed-point-free on $V, t$ cannot stabilize $V_{1}$ and $V_{2}$. For otherwise $t$ would centralize $W / C_{W}\left(V_{i}\right), i=1,2$, which implies that $[W, t] \leqslant C_{W}\left(V_{1}\right) \cap$ $C_{W}\left(V_{2}\right)=1$, a contradiction. So $t$ interchanges $V_{1}, V_{2}$. Also $s$ acts on $\left\{V_{1}, V_{2}\right\}$ as $s \in N(W)$. Consequently, $\bar{v} \in\langle\bar{s}, \bar{t}\rangle^{\prime}$ must fix each of $V_{1}$ and $V_{2}$. For $i=1,2$ let $\bar{F}_{i}=C_{\bar{E} \bar{E}^{s}}\left(V_{i}\right)$. Then $\bar{F}=\bar{F}_{1} \bar{F}_{2}=\bar{E} \bar{E}^{s}$. Write $J_{i} / R_{2}=V_{i}$. Then for $j_{1} \in J_{1}, j_{2} \in J_{2},\left[j_{1}, j_{2}\right]=\left[j_{1}, j_{2}^{g}\right]$ for each $g \in F_{1}$. So $\left[J_{2}, F_{1}\right] \leqslant$ $C\left(j_{1}\right)$, and, as $\left[J_{2}, F_{1}\right]$ covers $J_{2} / R_{2}$, we conclude that $\left[J_{1}, J_{2}\right]=1$.

Let $j_{1} \in J_{1}$. As $\left|J_{1} / R_{2}\right|=\left|R_{2}\right|=q^{2},\left[j_{1}, J_{1}\right]<R_{2}$. Suppose $q \neq 8$. Then $\bar{F}_{2}$ is irreducible on $J_{1} / R_{2}$, and $g \in F_{2}$ implies $\left[j_{1}, J_{1}\right]=\left[j_{1}^{8}, J_{1}\right]$. So here $\left[J_{1}, J_{1}\right]$ $<R_{2}$, and using the fact that $\left[J_{1}, J_{2}\right]=1$ and the action of $E_{00}$, we have $\left|J_{1}^{\prime}\right|=q$. If $q=8$ an easy Lie ring argument shows that $\left|J_{1}^{\prime}\right|=8$. Similarly, $\left|J_{2}^{\prime}\right|=q$. Setting $Y_{i}=\left[J_{i}, E\right]$ and using (6.7) we now have $U_{1}=Y_{1} \times Y_{2}$ with $t$ interchanging $Y_{1}$ and $Y_{2}$.

Since $t$ is an involution, $Y_{1} \cong Y_{2} \cong C_{U_{1}}(t)=T_{1}$. Hence $R_{2}=\Omega_{1}\left(U_{1}\right)$. The Krull-Schmidt theorem implies that $\left\{Y_{1} Z\left(U_{1}\right), Y_{2} Z\left(U_{1}\right)\right\}$ is invariant under $N_{G}\left(U_{1}\right)=N_{1}$. Also

$$
N_{1}=N_{N_{1}}\left(Y_{1} Z\left(U_{1}\right)\right)\langle t\rangle, \quad N_{0}=N_{N_{1}}\left(Y_{1} Z\left(U_{1}\right)\right) \triangleleft N_{1} .
$$

We choose $T_{3}$ and $s$ so that $\left\langle T_{3}, s\right\rangle \leqslant N_{0}$. Note that $s$ and $v$ normalize $\bar{E}_{00}$, $Y_{1}$, and $Y_{2}$. Also $\bar{F}=\bar{F}_{1} \times \bar{F}_{2}=\bar{E} \times \bar{E}^{s}$. Let $\bar{P}_{i}$ be a Sylow $p$-subgroup of $\bar{F}_{i}$, where $p=3$ if $q=8$ and $p$ a primitive divisor of $q+1$ if $q \neq 8$. Then $\bar{s}$ must centralize one of $\bar{P}_{1}$ and $\bar{P}_{2}$ and invert the other. Say $\bar{s}$ centralizes $\bar{P}_{1}$. Then $\bar{P}_{1}$ normalizes $\left[\bar{s}, V_{2}\right]$. If $\bar{s} \notin C\left(\bar{E}_{00}\right)$, then considering the Frobenius group $\left[\bar{E}_{00}, \bar{s}\right]\langle\bar{s}\rangle$, we conclude that $V_{2}$ is a free $\mathbf{F}_{2}(\langle\bar{s}\rangle)$-module. But then $\bar{P}_{1}$ cannot act on $\left[V_{2}, s\right]$. Therefore $\bar{s}$ centralizes $\bar{E}_{00}$ and, as $\bar{E}_{00} \bar{P}_{1}$ is irreducible on $V_{2}, \bar{s} \in C\left(V_{2}\right)$. This forces $\bar{s} \in C\left(\bar{F}_{1}\right)$.

Now consider the action of $\left\langle E_{00}, s, v\right\rangle$ on $R_{2}$ to get $\langle s, v\rangle \in C\left(R_{2}\right)$. Further, an application of the 3-subgroups lemma to $\langle s\rangle, Y_{2}$ and $F_{1}$ shows that $\left[s, Y_{2}\right]=1$.

We know that $\overline{\Omega_{1}\left(T_{3}\right)}\langle\bar{t}\rangle$ is a Sylow 2-subgroup of $C_{\bar{c}}(\bar{t})$, so the Sylow 2-subgroups of $C\langle t\rangle / U_{1}$ are dihedral or quasidihedral, where $C=N\left(U_{1}\right) \cap$ $C\left(R_{2}\right)$. Now $C \cap C\left(Y_{i}\right) \unlhd C$ for $i=1,2$, and from these facts we see that $\langle s, v\rangle U_{1} \in \operatorname{Syl}_{2}(C),\langle s, v\rangle U_{1} / U_{1}$ is klein, and $C / U_{1}$ has 2-complement 
$\left(\bar{s} \nsim \bar{v}\right.$ as $\left.\bar{s} \in C\left(V_{2}\right)\right)$. Let $C_{i}=C_{N_{1}}\left(Y_{i}\right)$ so that $F_{i} \leqslant C_{i}$. Then $\left[C_{1}, C_{2}\right]<$ $C\left(U_{1}\right)$ and $\left[\bar{C}_{1}, \bar{C}_{2}\right]=1$. As $\bar{C}_{2}=\bar{C}_{1}^{t}, \bar{C}_{1} \bar{C}_{2} \leqslant \bar{C}_{1} C_{\bar{C}}(\bar{t})$. This shows that $\bar{F}_{1} \bar{F}_{2}=O\left(\bar{C}_{1} \bar{C}_{2}\right) \leqslant \bar{N}_{1}$.

Say $q=8$. We claim that $U_{1}\langle s, v\rangle\langle t\rangle=S$. For $U_{1}\langle s, v\rangle\left\langle C\left(R_{2}\right)\right.$ implies that $t^{G} \cap U_{1}\langle s, v\rangle=1$, and the involutions in $U_{1}\langle s, v\rangle\langle t\rangle-U_{1}\langle s, v\rangle$ are in $U_{1} t \cup U_{1} t v$. As $\left(U_{1} t\right)^{s}=U_{1} t v$ and $t^{G} \cap U_{1} t=t^{U}{ }, U_{1}\langle s, v\rangle\langle t\rangle$ controls its fusion of conjugates of $t$, and the claim follows. Suppose $q \neq 8$; then $\bar{F}_{1}$ is irreducible on $Y_{2}$. As $\overline{N_{0}^{\prime}} \leqslant C\left(\bar{F}_{1}\right), \overline{N_{0}^{\prime}}$ induces a cyclic group on $V_{2}$. Similarly for $V_{1}$. So $\overline{N_{0}^{\prime}}$ is abelian of odd order and of rank at most 2. Since $C(t)$ covers $C(t) \cap\left(N_{0} / N_{0}^{\prime}\right), N_{0} / N_{0}^{\prime}$ has Sylow 2-subgroups of rank 2. It is now easy to check that $U_{1}$ is characteristic in $S \cap N_{1}$, so $S=S \cap N_{1} \in \operatorname{Syl}_{2}(G)$.

As $\Omega_{1}\left(S \cap N_{0}\right) \leqslant U_{1}\langle s, v\rangle \leqslant C\left(R_{2}\right), t^{G} \cap\left(S \cap N_{0}\right)=\varnothing$, and we apply transfer to obtain a subgroup $G_{0}$ of index 2 in $G$ with $U_{1}\langle v\rangle\left\langle G_{0}\right.$. Considering the action of $t$ on a Sylow 2-subgroup of $C_{G}(v)$, we see that

$$
v^{G} \cap U_{1}\langle s\rangle=v^{G} \cap U_{1}\langle v s\rangle=\varnothing .
$$

Again we can apply transfer to get $v \notin G^{\prime}$. But then $s^{t} \in s v U_{1}$ implies that $U_{1} \in \operatorname{Syl}_{2}\left(G^{\prime}\right)$, contradicting (3.3).

We have now completed the proof of the main theorem.

\section{BIBLIOGRAPHY}

1. M. Aschbacher, Tightly embedded subgroups of finite groups, J. Algebra 42 (1976), 85-101.

2. - Standard components of alternating type centralized by a 4-group (to appear).

3. M. Aschbacher and G. Seitz, On groups with a standard component of known type, Osaka J. Math. 13 (1976), 439-482.

4. S. Assa, Ph.D. Thesis, Ohio State University, 1973.

5. H. Bender, Transitive Gruppen gerader Ordnung in denen jede Involution genau einen Punkt festlasst, J. Algebra 17 (1971), 527-554.

6. U. Dempwolff, $A$ characterization of the Rudvalis simple group of order $2^{14} \cdot 3^{3} \cdot 5^{3} \cdot 7 \cdot 13$. 29 by the centralizer of noncentral involutions, J. Algebra 32 (1974), 53-88.

7. W. Feit and J. Thompson, Solvability of groups of odd order, Pacific J. Math. 13 (1963), 775-1029.

8. L. Finkelstein, Finite groups with a standard component of type Janko-Ree, J. Algebra 36 (1975), 416-426.

9. P. Fong and G. Seitz, Groups with $a(B, N)$-pair of rank 2. I, Invent. Math. 21 (1973), 1-57.

10. R. Gilman and D. Gorenstein, Finite groups with Sylow 2-subgroups of class 2, Trans. Amer. Math. Soc. 207 (1975), 1-126.

11. D. Goldschmidt, 2-fusion in finite groups, Ann. of Math. (2) 99 (1974), 70-117.

12. D. Gorenstein and K. Harada, Finite groups whose 2-subgroups are generated by at most 4 elements, Mem. Amer. Math. Soc., no. 147, 1974.

13. $331-406$. , A characterization of Janko's two new simple groups, J. Univ. Tokyo 16 (1970),

14. D. Gorenstein and J. Walter, The characterization of finite groups with dihedral Sylow 2-subgroups. I, II, III, J. Algebra 2 (1965), 85-151; 218-270; 354-393.

15. K. Harada, On finite groups having self-centralizing 2-subgroups of small order, J. Algebra 33 (1975), 144-160.

16. C. Hering, W. Kantor and G. Seitz, Finite groups with a split $(B, N)$-pair of rank 1, J. Algebra 20 (1972), 435-475. 
17. G. Higman, Suzuki 2-groups, Illinois J. Math. 7 (1963), 79-96.

18. D. R. Mason, On finite simple groups $G$ in which every element of $\mathcal{L}(G)$ is of Bender type, J. Algebra 40 (1976), 125-202.

19. M. O'Nan, Some evidence for the existence of a new simple group (to appear).

20. G. Seitz, Flag-transitive subgroups of Chevalley groups, Ann. of Math. (2) 97 (1973), 27-56.

21. F. L. Smith, $A$ general characterization of the Janko simple group $J_{2}$ (to appear).

22. M. Suzuki, On a class of doubly transitive groups, Ann. of Math. (2) 75 (1962), 105-145.

Department of Mathematics, University of Michigan, ANn Arbor, Michigan 48104

Department of Mathematics, University of Cambridge, Cambridge, England

Department of Mathematics, University of Oregon, Eugene, Oregon 97403 